$\mathrm{DTP} / 00 / 33$

hep-th/0004121

\title{
On the quantum reflection factor for the sinh-Gordon model with general boundary conditions
}

\author{
A. Chenaghlou 円 \\ Dept. of Mathematical Sciences, University of Durham, Durham DH1 3LE, UK
}

\begin{abstract}
The one loop quantum corrections to the classical reflection factor of the sinhGordon model are calculated partially for general boundary conditions. The model is studied under boundary conditions which are compatible with integrability, and in the framework of the conventional perturbation theory generalized to the affine Toda field theory. It is found that the general form of the related quantum corrections are hypergeometric functions.
\end{abstract}

\footnotetext{
${ }^{1}$ alireza. chenaghlou@durham.ac.uk
} 


\section{Introduction}

Affine Toda field theory [1, 2] is an integrable quantum field theory in two-dimensional Minkowski space-time which possesses remarkable properties and rich algebraic structure (for a review see [3]). This theory becomes more interesting [4 9] when it is restricted to a half-line. However, for most of the Toda theories corresponding to affine simply-laced algebras, the boundary conditions are limited to a finite number which preserve integrability. Corrigan et.al [4 6] have classified the boundary conditions which preserve classical integrability. However, there still remains much to be studied in relation to quantum integrability on the half-line. For models based on $a_{n}^{(1)}$ much is now known [由,10 12]. The simplest affine Toda theory, the sinh-Gordon model has been studied much more than other models in the context of integrable boundaries. This model is the only theory in the ade series of affine Toda field theory for which continuous boundary parameters are possible.

In recent years there has been considerable interest [12,14 18 in perturbative affine Toda field theory. The motivation behind this fact is that the boundary S-matrices

of the models are largely unknown. The most progress has been made for $a_{1}^{(1)}$ affine Toda field theory for which the general form of the boundary S-matrix has been found by Ghoshal [19]. In fact, the boundary bootstrap equations yield the boundary Smatrices up to some unknown parameters. The perturbation method not only provides an additional check of the results which come from the bootstrap technique, but also it could make a connection between the unknown parameters of the boundary S-matrices and the boundary parameters which are involved in the Lagrangian formulation of the theories.

Firstly, Ghoshal and Zamolodchikov [20] obtained the soliton reflection factors in the sine-Gordon model with a boundary consistent with integrability. Then, Ghoshal [19] using these results calculated the reflection factors of the soliton-anti-soliton bound states (the breathers) of the model. One of the interesting problem in the boundary sine(sinh)-Gordon model is to find the relation between the free parameters appearing in Ghoshal's formula and the boundary data appearing in the Lagrangian of the model. Corrigan [14 was the first to notice that the lightest breather reflection factor of the sine-Gordon model is identical to the reflection factor of the sinh-Gordon model after an analytic continuation in the coupling constant. In a recent paper Corrigan and Delius [13] studied the boundary breather states of the sinh-Gordon model on a halfline. They calculated the energy spectrum of the boundary states in two ways, by using the bootstrap equations then by using a WKB approximation. By comparing the results obtained by the two methods, they provided strong evidence for a conjectured relationship between the boundary parameters, the bulk coupling constant and the parameters appearing in the quantum reflection factor calculated by Ghoshal. They carried out the calculations in the special case when the boundary parameters are equal and the boundary condition preserve the $\phi \rightarrow-\phi$ symmetry of the bulk theory.

In [14 the quantum corrections up to $O\left(\beta^{2}\right)$ to the classical reflection factor of the sinh-Gordon model were found when the boundary parameters are equal. In this case, the static background configuration is $\phi=0$. If the boundary data are different then, the lowest energy solution will not be a trivial background. The correspond- 
ing perturbation theory involves complicated coupling constants and two-point Green function as well. Recently [21] the quantum reflection factor has been calculated in one loop order up to the first order in the difference of the two boundary parameters. The result [21] provide a further verification of Ghoshal's formula. This paper extends the results of [21], by calculating the quantum reflection factor for any value of the boundary parameters. It is found that most part of the related quantum corrections to the classical reflection factor may be expressed in terms of hypergeometric functions. This result and how it could relate to Ghoshal's formula is discussed in the conclusions.

\section{Boundary sinh-Gordon model}

The sinh-Gordon theory on the half-line is a massive scalar quantum field theory in $1+1$ dimension whose corresponding untwisted affine Kac-Moody algebra is $a_{1}^{(1)}$. The Lagrangian density of the theory is:

$$
\overline{\mathcal{L}}=\theta(-x) \mathcal{L}-\delta(x) \mathcal{B}
$$

Here, $\mathcal{L}$ is the bulk Lagrangian density of the model which is given by

$$
\begin{aligned}
\mathcal{L} & =\frac{1}{2} \partial_{\mu} \phi \partial^{\mu} \phi-V(\phi) \\
& =\frac{1}{2} \partial_{\mu} \phi \partial^{\mu} \phi-\frac{2 m^{2}}{\beta^{2}} \cosh (\beta \alpha \phi),
\end{aligned}
$$

where $\mathrm{m}$ and $\beta$ are a mass scale and a coupling constant of the theory. Moreover, the boundary potential $\mathcal{B}$ has the generic form 20]

$$
\mathcal{B}=\frac{m}{\beta^{2}}\left(\sigma_{0} e^{-\frac{\beta}{\sqrt{2}} \phi}+\sigma_{1} e^{\frac{\beta}{\sqrt{2}} \phi}\right) .
$$

In the above relation, the two real coefficients $\sigma_{0}$ and $\sigma_{1}$ are arbitrary and indicate [5,7] the degrees of freedom allowed at the boundary. In fact, Bowcock et.al [6] obtained some results about the form of the the boundary term via a generalized Lax pair when there is a boundary. For further discussion on the boundary parameters see [5, 7].

The sinh-Gordon model is integrable classically which means there are infinitely many independent conserved quantities $Q_{ \pm s}$ where s is an arbitrary odd integer. On the other hand, the model is integrable after quantizing which implies the S-matrix describing the n-particles scattering factorises into a product of two-particles scattering amplitudes. The S-matrix describing the elastic scattering of two sinh-Gordon particles of relative rapidity $\theta$ is conjectured to have the form [1, 22, 23]

$$
S(\theta)=-\frac{1}{(B)(2-B)}
$$

where we use the hyperbolic building blocks

$$
(x)=\frac{\sinh \left(\theta / 2+\frac{i \pi x}{4}\right)}{\sinh \left(\theta / 2-\frac{i \pi x}{4}\right)},
$$


and the quantity $\mathrm{B}$ is related to the coupling constant $\beta$ by $B=\frac{2 \beta^{2}}{4 \pi+\beta^{2}}$.

In order to maintain the integrability on the half-line, the boundary potential must satisfy the following equation

$$
\frac{\partial \phi}{\partial x}=-\frac{\partial \mathcal{B}}{\partial \phi} \quad \text { at } \quad x=0
$$

or

$$
\frac{\partial \phi}{\partial x}=-\frac{\sqrt{2} m}{\beta}\left(\sigma_{1} e^{\beta \phi / \sqrt{2}}-\sigma_{0} e^{-\beta \phi / \sqrt{2}}\right) \quad \text { at } x=0,
$$

where we use the normalization condition $\alpha^{2}=2$ which is customary in affine Toda field theory. In what follows the dimensional mass parameter $m$ will be taken to unity. It is also convenient to use $\sigma_{i}=\cos a_{i} \pi$. For the boundary sinh-Gordon model, besides to the two-particle S-matrix it is necessary to know the boundary S-matrix or reflection factor describing one particle reflection off the boundary. Firstly, Ghoshal and Zamolodchikov [20] calculated the soliton reflection factors for the sine-Gordon model by solving the boundary Yang-Baxter equation. Then, Ghoshal [19] calculated the soliton-antisoliton bound state reflection factor. He used the boundary bootstrap equations along with the result of reference [20]. The general form of the quantum reflection factor in sinh-Gordon model may be derived by regarding the lightest breather reflection factor of the sine-Gordon model [19], calculated by Ghoshal, and performing analytic continuation in the coupling constant to find

$$
K_{q}(\theta)=\frac{(1)(2-B / 2)(1+B / 2)}{\left(1-E\left(\sigma_{0}, \sigma_{1}, \beta\right)\right)\left(1+E\left(\sigma_{0}, \sigma_{1}, \beta\right)\right)\left(1-F\left(\sigma_{0}, \sigma_{1}, \beta\right)\right)\left(1+F\left(\sigma_{0}, \sigma_{1}, \beta\right)\right)} .
$$

Note the bulk reflection symmetry leads to $F=0$ or $E=0$ when $\sigma_{0}=\sigma_{1}$ (note only one of them vanishes). In fact, the exact form of the $E$ and $F$ is an open and hard problem. Recently Corrigan and Delius [13] obtained the function E in the special case when $\sigma_{0}=\sigma_{1}=\cos a \pi$ and $\frac{1}{2}<a<1$ as

$$
E=2 a(1-B / 2)
$$

They found the above formula by equating the results of the WKB approximation method and the bootstrap technique.

\section{Low order perturbation theory}

For affine Toda field theory the perturbative calculation is performed around the static background field configuration, so standard Feynman Rules may be used. By expanding

the bulk and boundary potentials in terms of the coupling constant $\beta$, the three and four point couplings can be deduced. We find for the sinh-Gordon theory

$$
C_{b u l k}^{(3)}=\frac{2 \sqrt{2}}{3} \beta \sinh \left(\sqrt{2} \beta \phi_{0}\right)
$$




$$
C_{\text {bulk }}^{(4)}=\frac{1}{3} \beta^{2} \cosh \left(\sqrt{2} \beta \phi_{0}\right),
$$

where $\phi_{0}$ represent the background solution to the equation of motion of the model and similarly

$$
\begin{aligned}
C_{\text {boundary }}^{(3)} & =\frac{\sqrt{2} \beta}{12}\left(\sigma_{1} e^{\beta \phi_{0} / \sqrt{2}}-\sigma_{0} e^{-\beta \phi_{0} / \sqrt{2}}\right) \\
C_{\text {boundary }}^{(4)} & =\frac{\beta^{2}}{48}\left(\sigma_{1} e^{\beta \phi_{0} / \sqrt{2}}+\sigma_{0} e^{-\beta \phi_{0} / \sqrt{2}}\right) .
\end{aligned}
$$

On the other hand, the static background field can be found through linear perturbation of the equation of motion and the boundary condition of the model [5, 14] to obtain

$$
e^{\beta \phi_{0} / \sqrt{2}}=\frac{1+e^{2\left(x-x_{0}\right)}}{1-e^{2\left(x-x_{0}\right)}},
$$

where the parameter $x_{0}$ is related to the boundary parameters by

$$
\operatorname{coth} x_{0}=\sqrt{\frac{1+\sigma_{0}}{1+\sigma_{1}}}
$$

So, the three and four point couplings corresponding to the bulk potential take the forms

$$
\begin{gathered}
C_{\text {bulk }}^{(3)}=\frac{4 \sqrt{2}}{3} \beta \cosh 2\left(x-x_{0}\right)\left(\operatorname{coth}^{2} 2\left(x-x_{0}\right)-1\right), \\
C_{\text {bulk }}^{(4)}=\frac{1}{3} \beta^{2}\left(2 \operatorname{coth}^{2} 2\left(x-x_{0}\right)-1\right) .
\end{gathered}
$$

In the same manner the three and four point couplings of the boundary are given by

$$
\begin{gathered}
C_{\text {boundary }}^{(3)}=\frac{\sqrt{2} \beta}{12}\left(\sigma_{1} \operatorname{coth} x_{0}-\sigma_{0} \tanh x_{0}\right), \\
C_{\text {boundary }}^{(4)}=\frac{\beta^{2}}{48}\left(\sigma_{1} \operatorname{coth} x_{0}+\sigma_{0} \tanh x_{0}\right) .
\end{gathered}
$$

The next step is to find the propagator for the theory. It has been shown [14] that the two-point Green function for the sinh-Gordon model on a half-line is

$$
\begin{array}{r}
G\left(x, t ; x^{\prime}, t^{\prime}\right)=\iint \frac{d \omega}{2 \pi} \frac{d k}{2 \pi} \frac{i e^{-i \omega\left(t-t^{\prime}\right)}}{\omega^{2}-k^{2}-4+i \rho}\left(f(k, x) f\left(-k, x^{\prime}\right) e^{i k\left(x-x^{\prime}\right)}\right. \\
\left.+K_{c} f(-k, x) f\left(-k, x^{\prime}\right) e^{-i k\left(x+x^{\prime}\right)}\right),
\end{array}
$$

where

$$
f(k, x)=\frac{i k-2 \operatorname{coth} 2\left(x-x_{0}\right)}{i k+2}
$$

and $K_{c}$ is the classical reflection factor of the model which is equal to

$$
K_{c}=\left(\frac{(i k)^{2}+2 i k \sqrt{1+\sigma_{0}} \sqrt{1+\sigma_{1}}+2\left(\sigma_{0}+\sigma_{1}\right)}{(i k)^{2}-2 i k \sqrt{1+\sigma_{0}} \sqrt{1+\sigma_{1}}+2\left(\sigma_{0}+\sigma_{1}\right)}\right)\left(\frac{i k-2}{i k+2}\right) .
$$


The classical reflection factor (3.13) can be derived from the quantum reflection factor (2.8) by considering the classical limit i.e. when $\beta \rightarrow 0$. Because, in this limit [5] $E=a_{0}+a_{1}$ and $F=a_{0}-a_{1}$. Now following the idea introduced by Kim 15 and developed by Corrigan [14], we may calculate the one loop quantum corrections to the classical reflection factor after perturbation calculation of the two-point function and then by finding the coefficient of $e^{-i k\left(x+x^{\prime}\right)}$ in the residue of the on-shell pole in the asymptotic region $x, x^{\prime} \rightarrow-\infty$.

In order to calculate the one loop $\left(O\left(\beta^{2}\right)\right)$ quantum corrections to the classical reflection factor, we use the standard perturbation theory which is generalized 14 17 to the affine Toda field theory on the half-line. In general, at $\left(O\left(\beta^{2}\right)\right)$ there are three basic kinds of Feynman diagrams contribute to the two-point propagator of affine Toda field theory . These are shown in figure 1. Moreover, by inspection of the forms of the three point and four point couplings which we have found, it is clear that all types of these diagrams are involved in our problem.

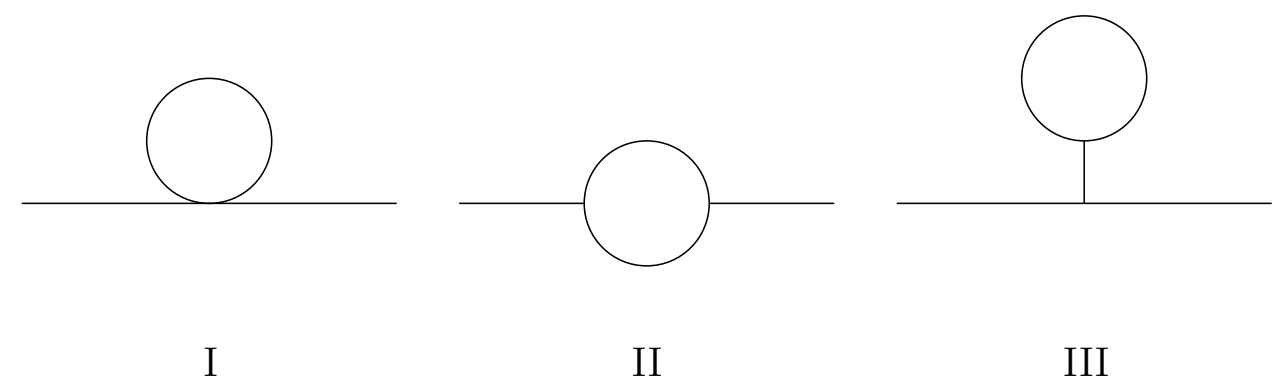

Figuer 1: Three basic Feynman diagrams in one loop order.

In fact, when the boundary parameters are not equal then, the calculations corresponding to the one loop order in the sinh-Gordon model are lengthy and intricate. In the following sections we try to compute the contributions of types I and III diagrams to the reflection factor. The remaining diagrams will be treated elsewhere. Meanwhile, it is instructive to start with type III.

\section{Type III diagram (boundary-boundary)}

In this section we shall calculate the contribution of the type III diagram to the reflection factor when both vertices are located at the boundary $x=0$. We are led to the following integral

$$
\begin{aligned}
& -\frac{\beta^{2}}{4}\left(\sigma_{1} \operatorname{coth} x_{0}-\sigma_{0} \tanh x_{0}\right)^{2} \\
& \times \iint d t d t^{\prime} G\left(x_{1}, t_{1} ; 0, t\right) G\left(0, t ; 0, t^{\prime}\right) G\left(0, t^{\prime} ; 0, t^{\prime}\right) G\left(0, t ; x_{2}, t_{2}\right)
\end{aligned}
$$

in which the combinatorial factor has been taken into account. 
Let us start by looking at the loop propagator $G\left(0, t^{\prime} ; 0, t^{\prime}\right)$ which is equal to

$$
\begin{aligned}
G\left(0, t^{\prime} ; 0, t^{\prime}\right)=i \iint \frac{d \omega^{\prime}}{2 \pi} \frac{d k^{\prime}}{2 \pi} & \frac{1}{\omega^{\prime 2}-k^{2}-4+i \rho} f\left(-k^{\prime}, 0\right) \\
& \times\left(f\left(k^{\prime}, 0\right)+K^{\prime}\left(k^{\prime}\right) f\left(-k^{\prime}, 0\right)\right),
\end{aligned}
$$

where

$$
f\left(k^{\prime}, 0\right)=\frac{i k^{\prime}+2 \operatorname{coth} 2 x_{0}}{i k^{\prime}+2}
$$

and $K^{\prime}\left(k^{\prime}\right)$ is the classical reflection factor (3.13). After some manipulation, we obtain

$$
\begin{aligned}
G\left(0, t^{\prime} ; 0, t^{\prime}\right)= & i \iint \frac{d \omega^{\prime}}{2 \pi} \frac{d k^{\prime}}{2 \pi} \frac{1}{\omega^{\prime 2}-k^{\prime 2}-4+i \rho} \\
& \times \frac{2 i k^{\prime}\left(i k^{\prime}-2 \operatorname{coth} 2 x_{0}\right)}{\left(i k^{\prime}\right)^{2}-2 i k^{\prime} \sqrt{1+\sigma_{0}} \sqrt{1+\sigma_{1}}+2\left(\sigma_{0}+\sigma_{1}\right)} .
\end{aligned}
$$

The above integral is clearly divergent however, the divergence can be removed by the infinite renormalization of the boundary term. In other words, considering the following relation

$$
\begin{aligned}
& \frac{2 i k^{\prime}\left(i k^{\prime}-2 \operatorname{coth} 2 x_{0}\right)}{\left(i k^{\prime}\right)^{2}-2 i k^{\prime} \sqrt{1+\sigma_{0}} \sqrt{1+\sigma_{1}}+2\left(\sigma_{0}+\sigma_{1}\right)} \\
& =2+4 \frac{i k^{\prime}\left(\sqrt{1+\sigma_{0}} \sqrt{1+\sigma_{1}}-\operatorname{coth} 2 x_{0}\right)-\left(\sigma_{0}+\sigma_{1}\right)}{\left(i k^{\prime}\right)^{2}-2 i k^{\prime} \sqrt{1+\sigma_{0}} \sqrt{1+\sigma_{1}}+2\left(\sigma_{0}+\sigma_{1}\right)},
\end{aligned}
$$

it is seen that a minimal subtraction of the divergent part can be made by adding an appropriate counter term to the boundary, replace the logarithmically divergent integral by the finite part. Hence,

$$
\begin{aligned}
G\left(0, t^{\prime} ; 0, t^{\prime}\right)= & 4 i \iint \frac{d \omega^{\prime}}{2 \pi} \frac{d k^{\prime}}{2 \pi} \frac{1}{\omega^{\prime 2}-k^{\prime 2}-4+i \rho} \\
& \times \frac{i k^{\prime}\left(\sqrt{1+\sigma_{0}} \sqrt{1+\sigma_{1}}-\operatorname{coth} 2 x_{0}\right)-\left(\sigma_{0}+\sigma_{1}\right)}{\left(i k^{\prime}\right)^{2}-2 i k^{\prime} \sqrt{1+\sigma_{0}} \sqrt{1+\sigma_{1}}+2\left(\sigma_{0}+\sigma_{1}\right)} .
\end{aligned}
$$

The integration over $\omega^{\prime}$ may be performed by closing the contour into the upper halfplane and collecting a pole at $\omega^{\prime}=\sqrt{k^{\prime 2}+4}$ so that

$$
G\left(0, t^{\prime} ; 0, t^{\prime}\right)=2 \int \frac{d k^{\prime}}{2 \pi} \frac{1}{\sqrt{k^{\prime 2}+4}} \frac{i k^{\prime}\left(\sqrt{1+\sigma_{0}} \sqrt{1+\sigma_{1}}-\operatorname{coth} 2 x_{0}\right)-\left(\sigma_{0}+\sigma_{1}\right)}{\left(i k^{\prime}\right)^{2}-2 i k^{\prime} \sqrt{1+\sigma_{0}} \sqrt{1+\sigma_{1}}+2\left(\sigma_{0}+\sigma_{1}\right)} .
$$

In order to integrate over $k^{\prime}$, as before, one chooses a contour in the upper half-plane, however due to the branch cut the contour has to run around the cut line. Moreover we assume that the roots of the denominator of the integrand i.e. $2 \cos \frac{\left(a_{0} \pm a_{1}\right) \pi}{2}$ are 
positive, otherwise we may close the contour in the lower half-plane. Therefore, (4.7) converts to

$$
4 \int_{2}^{\infty} \frac{d y}{2 \pi} \frac{1}{\sqrt{y^{2}-4}} \frac{y\left(\sqrt{1+\sigma_{0}} \sqrt{1+\sigma_{1}}-\operatorname{coth} 2 x_{0}\right)+\left(\sigma_{0}+\sigma_{1}\right)}{y^{2}+2 y \sqrt{1+\sigma_{0}} \sqrt{1+\sigma_{1}}+2\left(\sigma_{0}+\sigma_{1}\right)}
$$

and the above integral gives the following result

$$
G\left(0, t^{\prime} ; 0, t^{\prime}\right)=-\frac{a_{0}}{2} \cot a_{0} \pi-\frac{a_{1}}{2} \cot a_{1} \pi .
$$

Now it is convenient to calculate the time integral of the other middle propagator in (4.1) which is equal to

$$
\begin{aligned}
\int d t^{\prime} G\left(0, t ; 0, t^{\prime}\right)=\iint & \int t^{\prime} \frac{d \omega}{2 \pi} \frac{d k}{2 \pi} e^{-i \omega\left(t-t^{\prime}\right)} \frac{i}{\omega^{2}-k^{2}-4+i \rho} \\
& \times \frac{2 i k\left(i k-2 \operatorname{coth} 2 x_{0}\right)}{(i k)^{2}-2 i k \sqrt{1+\sigma_{0}} \sqrt{1+\sigma_{1}}+2\left(\sigma_{0}+\sigma_{1}\right)} .
\end{aligned}
$$

Clearly, in the boundary-boundary contribution (4.1), it is seen that the $t^{\prime}$ dependence is involved only in the propagator $G\left(0, t ; 0, t^{\prime}\right)$ so, the integration over $t^{\prime}$ gives us a Dirac delta function which means we must substitute zero for $\omega$ and hence

$$
\int d t^{\prime} G\left(0, t ; 0, t^{\prime}\right)=\int \frac{d k}{2 \pi}\left(\frac{i}{-k^{2}-4}\right)\left(\frac{2 i k\left(i k-2 \operatorname{coth} 2 x_{0}\right)}{(i k)^{2}-2 i k \sqrt{1+\sigma_{0}} \sqrt{1+\sigma_{1}}+2\left(\sigma_{0}+\sigma_{1}\right)}\right) .
$$

As we mentioned before, throughout this paper we assume that the roots of $P(k)=$ $(i k)^{2}-2 i k \sqrt{1+\sigma_{0}} \sqrt{1+\sigma_{1}}+2\left(\sigma_{0}+\sigma_{1}\right)$ which are equal to $2 \cos \frac{\left(a_{0} \pm a_{1}\right) \pi}{2}$ are positive, so the $P(k)$ has no pole in the upper half-plane. Obviously, if the roots are negative then we can choose the contour in the lower half-plane in which no pole is inserted. Therefore,

$$
\int d t^{\prime} G\left(0, t ; 0, t^{\prime}\right)=\frac{i\left(1+\operatorname{coth} 2 x_{0}\right)}{2+2 \sqrt{1+\sigma_{0}} \sqrt{1+\sigma_{1}}+\left(\sigma_{0}+\sigma_{1}\right)}
$$

and by substituting $\sigma_{0}=\cos a_{0} \pi$ and $\sigma_{1}=\cos a_{1} \pi$, we obtain

$$
\int d t^{\prime} G\left(0, t ; 0, t^{\prime}\right)=-\frac{i}{4 \cos \frac{a_{0} \pi}{2} \cos \frac{a_{1} \pi}{2}} .
$$

Up to now, the boundary-boundary contribution has the form

$$
\begin{aligned}
& -\frac{i \beta^{2}}{32} \frac{\left(\sigma_{1} \operatorname{coth} x_{0}-\sigma_{0} \tanh x_{0}\right)^{2}\left(a_{0} \cot a_{0} \pi+a_{1} \cot a_{1} \pi\right)}{\cos \frac{a_{0} \pi}{2} \cos \frac{a_{1} \pi}{2}} \\
& \times \int d t \iint \frac{d \omega_{1}}{2 \pi} \frac{d k_{1}}{2 \pi} \frac{i e^{-i \omega_{1}\left(t_{1}-t\right)}}{\omega_{1}^{2}-k_{1}^{2}-4+i \rho}\left(f\left(k_{1}, x_{1}\right) f\left(-k_{1}, 0\right) e^{i k_{1} x_{1}}\right. \\
& \left.+K_{1}\left(k_{1}\right) f\left(-k_{1}, x_{1}\right) f\left(-k_{1}, 0\right) e^{-i k_{1} x_{1}}\right) \\
& \times \iint \frac{d \omega_{2}}{2 \pi} \frac{d k_{2}}{2 \pi} \frac{i e^{-i \omega_{2}\left(t-t_{2}\right)}}{\omega_{2}^{2}-k_{2}^{2}-4+i \rho}\left(f\left(k_{2}, x_{2}\right) f\left(-k_{2}, 0\right) e^{-i k_{2} x_{2}}\right. \\
& \left.+K_{2}\left(k_{2}\right) f\left(-k_{2}, x_{2}\right) f\left(-k_{2}, 0\right) e^{-i k_{2} x_{2}}\right) .
\end{aligned}
$$


First of all, it is necessary to perform the transformation $k_{1} \rightarrow-k_{1}$ in the first term of the first propagator. Secondly, integration over $t$ ensures energy conservation at the interaction vertex and generates a Dirac delta function because of which we can set $\omega_{1}=\omega_{2}$. Moreover, it is better to define a new function as

$$
A(k, x)=f(-k, x) f(k, 0)+K(k) f(-k, x) f(-k, 0)
$$

or, in an expanded form,

$$
\begin{aligned}
A(k, x)= & \frac{i k+2 \operatorname{coth} 2 x_{0}}{i k+2} \frac{i k+2 \operatorname{coth} 2\left(x-x_{0}\right)}{i k-2} \\
& +\frac{\left(i k+2 \cos \frac{\left(a_{0}+a_{1}\right) \pi}{2}\right)\left(i k+2 \cos \frac{\left(a_{0}-a_{1}\right) \pi}{2}\right)}{\left(i k-2 \cos \frac{\left(a_{0}+a_{1}\right) \pi}{2}\right)\left(i k-2 \cos \frac{\left(a_{0}-a_{1}\right) \pi}{2}\right)} \\
& \times \frac{i k-2 \operatorname{coth} 2 x_{0}}{i k-2} \frac{i k+2 \operatorname{coth} 2\left(x-x_{0}\right)}{i k+2},
\end{aligned}
$$

then, the expression (4.14) reduces to

$$
\begin{aligned}
& -\frac{i \beta^{2}}{32} \frac{\left(\sigma_{1} \operatorname{coth} x_{0}-\sigma_{0} \tanh x_{0}\right)^{2}\left(a_{0} \cot a_{0} \pi+a_{1} \cot a_{1} \pi\right)}{\cos \frac{a_{0} \pi}{2} \cos \frac{a_{1} \pi}{2}} \\
& \times \iiint \frac{d \omega_{1}}{2 \pi} \frac{d k_{1}}{2 \pi} \frac{d k_{2}}{2 \pi} e^{-i \omega_{1}\left(t_{1}-t_{2}\right)} \frac{i}{\omega_{1}^{2}-k_{1}^{2}-4+i \rho} \frac{i}{\omega_{1}^{2}-k_{2}^{2}-4+i \rho} e^{-i k_{1} x_{1}} e^{-i k_{2} x_{2}} \\
& \times A\left(k_{1}, x_{1}\right) A\left(k_{2}, x_{2}\right) .
\end{aligned}
$$

Obviously, what we need to do next is to integrate over the momenta $k_{1}$ and $k_{2}$ and this may be achieved by closing the contours in the upper half-plane and considering the poles at $\hat{k}_{1}=k_{1}=k_{2}=\sqrt{\omega_{1}^{2}-4}$. Note, the additional poles due to functions $A\left(k_{1}, x_{1}\right)$ and $A\left(k_{2}, x_{2}\right)$ are not important because their contributions will be exponentially damped as $x_{1}, x_{2} \rightarrow-\infty$. Therefore, the boundary-boundary contribution is

$$
\begin{aligned}
& -\frac{i \beta^{2}}{32} \frac{\left(\sigma_{1} \operatorname{coth} x_{0}-\sigma_{0} \tanh x_{0}\right)^{2}\left(a_{0} \cot a_{0} \pi+a_{1} \cot a_{1} \pi\right)}{\cos \frac{a_{0} \pi}{2} \cos \frac{a_{1} \pi}{2}} \\
& \times \int \frac{d \omega_{1}}{2 \pi} e^{-i \omega_{1}\left(t_{1}-t_{2}\right)} e^{-i \hat{k}_{1}\left(x_{1}+x_{2}\right)} \frac{1}{\left(2 \hat{k}_{1}\right)^{2}} A\left(\hat{k}_{1}, x_{1}\right) A\left(\hat{k}_{2}, x_{2}\right) .
\end{aligned}
$$

Now recall the definition of the quantum reflection factor as the coefficient of $e^{-i k\left(x+x^{\prime}\right)}$ in the two-point Green function in the residue of the on-shell pole when $x, x^{\prime} \rightarrow-\infty$. Thus, the correction to the reflection factor from the type III (boundaryboundary) piece is

$$
\begin{aligned}
& -\frac{i \beta^{2}}{32} \frac{\left(\sigma_{1} \operatorname{coth} x_{0}-\sigma_{0} \tanh x_{0}\right)^{2}\left(a_{0} \cot a_{0} \pi+a_{1} \cot a_{1} \pi\right)}{\cos \frac{a_{0} \pi}{2} \cos \frac{a_{1} \pi}{2}} \\
& \times \frac{1}{2 \hat{k}_{1}}\left(\frac{\left(i \hat{k}_{1}+2 \operatorname{coth} 2 x_{0}\right)^{2}}{\left(i \hat{k}_{1}+2\right)^{2}}+2 K\left(\hat{k}_{1}\right) \frac{\left(i \hat{k}_{1}+2 \operatorname{coth} 2 x_{0}\right)\left(i \hat{k}_{1}-2 \operatorname{coth} 2 x_{0}\right)}{\left(i \hat{k}_{1}+2\right)^{2}}\right. \\
& \left.+K^{2}\left(\hat{k}_{1}\right) \frac{\left(i \hat{k}_{1}-2 \operatorname{coth} 2 x_{0}\right)^{2}}{\left(i \hat{k}_{1}+2\right)^{2}}\right)
\end{aligned}
$$




\section{Type III (boundary-bulk)}

This section deals with the determination of the contribution of the type III Feynman diagram to the classical reflection factor when one of the vertices corresponding to the loop is situated at the boundary and the other one is inside the bulk region. It is evident that in this case we have to take into account the bulk three point coupling $C_{b u l k}^{(3)}$ in

the corresponding vertex as well as the boundary three point coupling $C_{\text {boundary }}^{(3)}$ in the other vertex. Meanwhile, the combinatorial factor associated with the related Feynman diagram must be considered as a coefficient factor. Therefore, the contribution of the type III (boundary-bulk) to the reflection factor may be written as

$$
\begin{aligned}
-2 \beta^{2}\left(\sigma_{1} \operatorname{coth} x_{0}-\sigma_{0} \tanh x_{0}\right) & \iiint d t d t^{\prime} d x G\left(x_{1}, t_{1} ; x, t\right) G\left(x, t ; 0, t^{\prime}\right) \\
& \times G\left(0, t^{\prime} ; 0, t^{\prime}\right) G\left(x, t ; x_{2}, t_{2}\right) \sinh \left(\sqrt{2} \beta \phi_{0}\right) .
\end{aligned}
$$

The propagator $G\left(0, t^{\prime} ; 0, t^{\prime}\right)$ corresponding to the loop has been found in the previous section and is given by

$$
G\left(0, t^{\prime} ; 0, t^{\prime}\right)=-\frac{a_{0}}{2} \cot a_{0} \pi-\frac{a_{1}}{2} \cot a_{1} \pi
$$

The calculation of the other middle propagator i.e. $G\left(x, t ; 0, t^{\prime}\right)$ is the next step and clearly, the $t^{\prime}$ dependence in (5.1) is included only in this propagator. Hence, it is convenient to compute the following relation

$$
\begin{array}{r}
\int d t^{\prime} G\left(x, t ; 0, t^{\prime}\right)=\int d t^{\prime} \iint \frac{d \omega}{2 \pi} \frac{d k}{2 \pi} e^{-i \omega\left(t-t^{\prime}\right)} \frac{i}{\omega^{2}-k^{2}-4+i \rho}\left(f(k, x) f(-k, 0) e^{i k x}\right. \\
\left.+K(k) f(-k, x) f(-k, 0) e^{-i k x}\right) .(5.3)
\end{array}
$$

Integrating over $t^{\prime}$ generates a Dirac delta function and so,

$$
\begin{array}{r}
\int d t^{\prime} G\left(x, t ; 0, t^{\prime}\right)=i \int \frac{d k}{2 \pi} \frac{1}{\left(-k^{2}-4+i \rho\right)}\left(f(k, x) f(-k, 0) e^{i k x}\right. \\
\left.+K(k) f(-k, x) f(-k, 0) e^{-i k x}\right)
\end{array}
$$

and the residue theorem gives

$$
\int d t^{\prime} G\left(x, t ; 0, t^{\prime}\right)=\frac{i e^{2 x}}{8}\left(c_{0}+c_{1} \operatorname{coth} 2\left(x-x_{0}\right)\right),
$$

where

$$
c_{0}=-c_{1}=-\left(1+\tan ^{2} \frac{\left(a_{0}+a_{1}\right) \pi}{4}\right)\left(1+\tan ^{2} \frac{\left(a_{0}-a_{1}\right) \pi}{4}\right) .
$$

In order to check the above result, if we set $x=0$ in (5.5) then it will be equal to (4.13). 
Up to now the type III (boundary-bulk) contribution take the following form, of course, after integrating over $t$ :

$$
\begin{aligned}
& \beta^{2} c_{0}\left(\sigma_{1} \operatorname{coth} x_{0}-\sigma_{0} \tanh x_{0}\right)\left(a_{0} \cot a_{0} \pi+a_{1} \cot a_{1} \pi\right) \\
& \times \int_{-\infty}^{0} d x \iint \frac{d \omega_{1}}{2 \pi} \frac{d k_{1}}{2 \pi} e^{-i \omega_{1}\left(t_{1}-t_{2}\right)} \frac{i}{\omega_{1}^{2}-k_{1}^{2}-4+i \rho}\left(f\left(k_{1}, x_{1}\right) f\left(-k_{1}, x\right) e^{i k_{1}\left(x_{1}-x\right)}\right. \\
& \left.+K_{1}\left(k_{1}\right) f\left(-k_{1}, x_{1}\right) f\left(-k_{1}, x\right) e^{-i k_{1}\left(x+x_{1}\right)}\right) \\
& \times\left\{\frac{i e^{2 x}}{8}\left(1-\operatorname{coth} 2\left(x-x_{0}\right)\right) \sinh \left(\sqrt{2} \beta \phi_{0}\right)\right\} \\
& \times \int \frac{d k_{2}}{2 \pi} \frac{i}{\omega_{1}^{2}-k_{2}^{2}-4+i \rho}\left(f\left(k_{2}, x\right) f\left(-k_{2}, x_{2}\right) e^{i k_{2}\left(x-x_{2}\right)}\right. \\
& \left.+K_{2}\left(k_{2}\right) f\left(-k_{2}, x\right) f\left(-k_{2}, x_{2}\right) e^{-i k_{2}\left(x+x_{2}\right)}\right) .
\end{aligned}
$$

By multiplying the two propagators in (5.7) by each other, it is clear that one obtains four pole pieces and, as far as the integration over $x$ is concerned, if we do the integration over $x$ on one of them then, obviously the other three pole pieces could be done in the same manner. Hence, in what follows it is sufficient to treat only one of them and, meanwhile, keeping those terms which are functions of $x$, we are led to the following complicated integral

$$
\int_{-\infty}^{0} d x \exp \left\{2+i\left(k_{2}-k_{1}\right) x\right\} f\left(-k_{1}, x\right) f\left(k_{2}, x\right) \sinh \left(\sqrt{2} \beta \phi_{0}\right)\left(1-\operatorname{coth} 2\left(x-x_{0}\right)\right) .
$$

After some substitutions and collecting together powers of $\operatorname{coth} 2\left(x-x_{0}\right)$ we obtain

$$
\begin{array}{r}
\frac{1}{\left(i k_{1}-2\right)\left(i k_{2}+2\right)} \int_{-\infty}^{0} d x \exp \left\{2+i\left(k_{2}-k_{1}\right) x\right\} \sinh \left(\sqrt{2} \beta \phi_{0}\right)\left(-k_{1} k_{2}\right. \\
+\left(2 i k_{2}-2 i k_{1}+k_{1} k_{2}\right) \operatorname{coth} 2\left(x-x_{0}\right)+\left(2 i k_{1}-\right. \\
\left.\quad 2 i k_{2}-4\right) \operatorname{coth}^{2} 2\left(x-x_{0}\right) \\
\left.+4 \operatorname{coth}^{3} 2\left(x-x_{0}\right)\right) \cdot
\end{array}
$$

It is clear that in order to solve the above integral, it is necessary to manipulate the following integrals

$$
\int_{-\infty}^{0} d x \exp \left\{2+i\left(k_{2}-k_{1}\right) x\right\} \sinh \left(\sqrt{2} \beta \phi_{0}\right) \operatorname{coth}^{n} 2\left(x-x_{0}\right),
$$

where, $n=0,1,2,3$.

In fact in Appendix A, we have found the integrals (5.10) and the solutions of them are expressed in terms of hypergeometric functions. So, using the formulae in Appendix A and simplifying, we find that $\int$ in $(5.9)$ can be rewritten

$$
\mathcal{F}\left(k_{1}, k_{2}\right)=-\frac{3 k_{1} k_{2}+4 i k_{2}-4 i k_{1}+8}{3} \frac{1}{\sinh 2 x_{0}}-\frac{3 k_{1} k_{2}+6 i k_{2}-6 i k_{1}+13}{6} \frac{\cosh 2 x_{0}}{\sinh ^{2} 2 x_{0}}
$$




$$
\begin{aligned}
& -\frac{i k_{2}-i k_{1}+2}{3} \frac{\cosh ^{2} 2 x_{0}+1}{\sinh ^{3} 2 x_{0}}-\frac{1}{6} \frac{\cosh ^{3} 2 x_{0}+5 \cosh 2 x_{0}}{\sinh ^{4} 2 x_{0}} \\
& -\frac{12 i k_{1} k_{2}-16 k_{2}+16 k_{1}+40 i-\left(k_{2}-k_{1}\right)\left(9 k_{1} k_{2}+16 i k_{2}-16 i k_{1}+34\right)}{3\left(k_{2}-k_{1}-4 i\right)} \\
& \quad \times e^{-2 x_{0}} F\left(1, \frac{i}{4}\left(k_{2}-k_{1}\right)+1, \frac{i}{4}\left(k_{2}-k_{1}\right)+2, e^{-4 x_{0}}\right) \\
& -\frac{12 i k_{1} k_{2}-48 k_{2}+48 k_{1}+136 i-\left(k_{2}-k_{1}\right)\left(6 k_{1} k_{2}+28 i k_{2}-28 i k_{1}+84\right)}{3\left(k_{2}-k_{1}-8 i\right)} \\
& \times e^{-6 x_{0}} F\left(2, \frac{i}{4}\left(k_{2}-k_{1}\right)+2, \frac{i}{4}\left(k_{2}-k_{1}\right)+3, e^{-4 x_{0}}\right) \\
& +\frac{32 k_{2}-32 k_{1}-192 i+\left(k_{2}-k_{1}\right)\left(16 i k_{2}-16 i k_{1}+104\right)}{3\left(k_{2}-k_{1}-12 i\right)} \\
& \times \frac{16 k_{2}-16 k_{1}-32 i}{\left(k_{2}-k_{1}-16 i\right)} F\left(3, \frac{i}{4}\left(k_{2}-k_{1}\right)+3, \frac{i}{4}\left(k_{2}-k_{1}\right)+4, e^{-4 x_{0}}\right) \\
& \times e^{-14 x_{0}} F\left(4, \frac{i}{4}\left(k_{2}-k_{1}\right)+4, \frac{i}{4}\left(k_{2}-k_{1}\right)+5, e^{-4 x_{0}}\right) .
\end{aligned}
$$

Now regarding (5.7), after doing the transformation $k_{1} \rightarrow-k_{1}$ in the first term of the first propagator, all that remains is to integrate over the momenta $k_{1}$ and $k_{2}$ and this can be achieved by closing the contours in the upper half-plane and considering poles at $\hat{k}_{1}=k_{1}=k_{2}=\sqrt{\omega_{1}^{2}-4}$. The extra poles in the four functions $\mathcal{F}\left( \pm k_{1}, \pm k_{2}\right)$ are not important because their contributions will be discounted when $x_{1}$ and $x_{2}$ go to $-\infty$.

Let us write down the type III (boundary-bulk) contribution to the reflection factor

$$
\begin{aligned}
& \frac{\beta^{2}}{2} \frac{\tan \frac{\left(a_{0}+a_{1}\right) \pi}{4} \tan \frac{\left(a_{0}-a_{1}\right) \pi}{4}}{\cos \frac{a_{0} \pi}{2} \cos \frac{a_{1} \pi}{2}}\left(a_{0} \cot a_{0} \pi+a_{1} \cot a_{1} \pi\right) \\
& \times \int \frac{d \omega_{1}}{2 \pi} e^{-i \omega_{1}\left(t_{1}-t_{2}\right)} e^{-i \hat{k}_{1}\left(x_{1}+x_{2}\right)} \frac{1}{\left(2 \hat{k}_{1}\right)^{2}} \frac{i \hat{k}_{1}+2 \operatorname{coth} 2\left(x_{1}-x_{0}\right)}{i \hat{k}_{1}-2} \frac{i \hat{k}_{1}+2 \operatorname{coth} 2\left(x_{2}-x_{0}\right)}{i \hat{k}_{1}-2} \\
& \times\left\{\frac{i}{\left(i \hat{k}_{1}+2\right)^{2}} \mathcal{F}\left(-\hat{k}_{1}, \hat{k}_{1}\right)-\frac{i}{\left(i \hat{k}_{1}+2\right)\left(i \hat{k}_{1}-2\right)} K_{1}\left(\hat{k}_{1}\right) \mathcal{F}\left(\hat{k}_{1}, \hat{k}_{1}\right)\right. \\
& \left.\quad-\frac{i}{\left(i \hat{k}_{1}+2\right)\left(i \hat{k}_{1}-2\right)} K_{1}\left(\hat{k}_{1}\right) \mathcal{F}\left(-\hat{k}_{1},-\hat{k}_{1}\right)+\frac{i}{\left(i \hat{k}_{1}-2\right)^{2}} K_{1}^{2}\left(\hat{k}_{1}\right) \mathcal{F}\left(\hat{k}_{1},-\hat{k}_{1}\right)\right\}
\end{aligned}
$$

Now looking at the function $\mathcal{F}\left(k_{1}, k_{2}\right)$ given by (5.11), let us show the detailed forms of $\mathcal{F}\left(-\hat{k}_{1}, \hat{k}_{1}\right), \mathcal{F}\left(\hat{k}_{1}, \hat{k}_{1}\right), \mathcal{F}\left(-\hat{k}_{1},-\hat{k}_{1}\right)$ and $\mathcal{F}\left(\hat{k}_{1},-\hat{k}_{1}\right)$. In fact,

$$
\begin{aligned}
\mathcal{F}\left(\hat{k}_{1}, \hat{k}_{1}\right)= & -\frac{3 \hat{k}_{1}^{2}+8}{3} \frac{1}{\sinh 2 x_{0}}+\frac{3 \hat{k}_{1}^{2}+13}{6} \frac{\cosh 2 x_{0}}{\sinh ^{2} 2 x_{0}} \\
& -\frac{2}{3} \frac{\cosh ^{2} 2 x_{0}+1}{\sinh ^{3} 2 x_{0}}-\frac{1}{6} \frac{\cosh ^{3} 2 x_{0}+5 \cosh 2 x_{0}}{\sinh ^{4} 2 x_{0}}
\end{aligned}
$$




$$
\begin{aligned}
& +\frac{3 \hat{k}_{1}^{2}+10}{3} e^{-2 x_{0}} F\left(1,1,2, e^{-4 x_{0}}\right) \\
& +\frac{3 \hat{k}_{1}^{2}+34}{6} e^{-6 x_{0}} F\left(2,2,3, e^{-4 x_{0}}\right) \\
& +\frac{16}{3} e^{-10 x_{0}} F\left(3,3,4, e^{-4 x_{0}}\right) \\
& +2 e^{-14 x_{0}} F\left(4,4,5, e^{-4 x_{0}}\right) .
\end{aligned}
$$

It can be easily verified that

$$
\mathcal{F}\left(-\hat{k}_{1},-\hat{k}_{1}\right)=\mathcal{F}\left(\hat{k}_{1}, \hat{k}_{1}\right)
$$

and

$$
\begin{aligned}
\mathcal{F}\left(-\hat{k}_{1}, \hat{k}_{1}\right)= & \frac{3 \hat{k}_{1}^{2}-8 i \hat{k}_{1}-8}{3} \frac{1}{\sinh 2 x_{0}}+\frac{3 \hat{k}_{1}^{2}-12 i \hat{k}_{1}-13}{6} \frac{\cosh 2 x_{0}}{\sinh ^{2} 2 x_{0}} \\
& -\frac{2 i \hat{k}_{1}+2}{3} \frac{\cosh ^{2} 2 x_{0}}{\sinh ^{3} 2 x_{0}}-\frac{1}{6} \frac{\cosh ^{3} 2 x_{0}+5 \cosh 2 x_{0}}{\sinh ^{4} 2 x_{0}} \\
& -\frac{9 \hat{k}_{1}^{3}-38 i \hat{k}_{1}^{2}-50 \hat{k}_{1}+20 i}{3\left(\hat{k}_{1}-2 i\right)} e^{-2 x_{0}} F\left(1, \frac{i}{2} \hat{k}_{1}+1, \frac{i}{2} \hat{k}_{1}+2, e^{-4 x_{0}}\right) \\
& -\frac{6 \hat{k}_{1}^{3}-62 i \hat{k}_{1}^{2}-132 \hat{k}_{1}+68 i}{3\left(\hat{k}_{1}-4 i\right)} e^{-6 x_{0}} F\left(2, \frac{i}{2} \hat{k}_{1}+2, \frac{i}{2} \hat{k}_{1}+3, e^{-4 x_{0}}\right) \\
& +\frac{32 i \hat{k}_{1}^{2}+136 \hat{k}_{1}-96 i}{3\left(\hat{k}_{1}-6 i\right)} e^{-10 x_{0}} F\left(3, \frac{i}{2} \hat{k}_{1}+3, \frac{i}{2} \hat{k}_{1}+4, e^{-4 x_{0}}\right) \\
& +\frac{16 \hat{k}_{1}-16 i}{\left(\hat{k}_{1}-8 i\right)} e^{-14 x_{0}} F\left(4, \frac{i}{2} \hat{k}_{1}+4, \frac{i}{2} \hat{k}_{1}+5, e^{-4 x_{0}}\right) .
\end{aligned}
$$

Finally $\mathcal{F}\left(\hat{k}_{1},-\hat{k}_{1}\right)$ can be obtained from $\mathcal{F}\left(-\hat{k}_{1}, \hat{k}_{1}\right)$ after setting $\hat{k}_{1} \rightarrow-\hat{k}_{1}$.

\section{Type III(bulk-boundary)}

In this section we study the quantum correction to the classical reflection factor due to the contribution of the type III Feynman diagram, when the vertex associated with the loop is located at the bulk region and the other vertex coincides with the boundary. The associated contribution is given by

$$
\begin{array}{r}
\mathcal{C}=-2 \beta^{2}\left(\sigma_{1} \operatorname{coth} x_{0}-\sigma_{0} \tanh x_{0}\right) \iiint d t d t^{\prime} d x^{\prime} G\left(x_{1}, t_{1} ; 0, t\right) G\left(0, t ; x^{\prime}, t^{\prime}\right) \\
\times G\left(x^{\prime}, t^{\prime} ; x^{\prime}, t^{\prime}\right) G\left(0, t ; x_{2}, t_{2}\right) \sinh \left(\sqrt{2} \beta \phi_{0}\right) .
\end{array}
$$

The following relation which is some part of the contribution (6.1), can be derived independently from the remaining part

$$
\mathcal{C}_{1}=\int d t G\left(x_{1}, t_{1} ; 0, t\right) G\left(0, t ; x_{2}, t_{2}\right)
$$


or

$$
\begin{array}{r}
\mathcal{C}_{1}=\int d t \iint \frac{d \omega_{1}}{2 \pi} \frac{d k_{1}}{2 \pi} e^{-i \omega_{1}\left(t_{1}-t\right)} \frac{i}{\omega_{1}^{2}-k_{1}^{2}-4+i \rho}\left(f\left(k_{1}, x_{1}\right) f\left(-k_{1}, 0\right) e^{i k_{1} x_{1}}\right. \\
\left.+K_{1}\left(k_{1}\right) f\left(-k_{1}, x_{1}\right) f\left(-k_{1}, 0\right) e^{-i k_{1} x_{1}}\right) \\
\times \iint \frac{d \omega_{2}}{2 \pi} \frac{d k_{2}}{2 \pi} e^{-i \omega_{2}\left(t-t_{2}\right)} \frac{i}{\omega_{2}^{2}-k_{2}^{2}-4+i \rho}\left(f\left(k_{2}, 0\right) f\left(-k_{2}, x_{2}\right) e^{-i k_{2} x_{2}}\right. \\
\left.+K_{2}\left(k_{2}\right) f\left(-k_{2}, 0\right) f\left(-k_{2}, x_{2}\right) e^{-i k_{2} x_{2}}\right) .
\end{array}
$$

First of all, it is necessary to set $k_{1} \rightarrow-k_{1}$ in the first term of the first propagator. Secondly, integration over $t$ leads to the substitution of $\omega_{2}=\omega_{1}$. Finally integration over the momenta $k_{1}$ and $k_{2}$, as before, may be done immediately by closing the contour in the upper half-plane and looking at the poles at $\hat{k}_{1}=k_{1}=k_{2}=\sqrt{\omega_{1}^{2}-4}$ and ignoring all the other poles as their contributions vanish rapidly as $x_{1}, x_{2} \rightarrow-\infty$. Therefore,

$$
\mathcal{C}_{1}=\int \frac{d \omega_{1}}{2 \pi} e^{-i \omega_{1}\left(t_{1}-t_{2}\right)} e^{-i \hat{k}_{1}\left(x_{1}+x_{2}\right)} \frac{1}{\left(2 \hat{k}_{1}\right)^{2}} A\left(\hat{k}_{1}, x_{1}\right) A\left(\hat{k}_{1}, x_{2}\right),
$$

where

$$
A\left(\hat{k}_{1}, x_{1}\right)=f\left(-\hat{k}_{1}, x_{1}\right) f\left(\hat{k}_{1}, 0\right)+K\left(\hat{k}_{1}\right) f\left(-\hat{k}_{1}, x_{1}\right) f\left(-\hat{k}_{1}, 0\right) .
$$

So, our next problem is to calculate the following integral which is the remaining part of the contribution.

$$
\iint d t^{\prime} d x^{\prime} G\left(0, t ; x^{\prime}, t^{\prime}\right) G\left(x^{\prime}, t^{\prime} ; x^{\prime}, t^{\prime}\right) \sinh \left(\sqrt{2} \beta \phi_{0}\right) .
$$

Obviously, this part will be appeared as a constant and it must be multiplied by (6.4). Clearly, the time variable $t^{\prime}$ appears only in one of the propagator i.e. in $G\left(0, t ; x^{\prime}, t^{\prime}\right)$. On the other hand, this propagator along with integration over $t^{\prime}$ has been obtained in the previous section. Hence,

$$
\int d t^{\prime} G\left(0, t ; x^{\prime}, t^{\prime}\right)=\frac{i}{8} e^{2 x^{\prime}} c_{0}\left(1-\operatorname{coth} 2\left(x^{\prime}-x_{0}\right)\right),
$$

where

$$
c_{0}=-\left(1+\tan ^{2} \frac{\left(a_{0}+a_{1}\right) \pi}{4}\right)\left(1+\tan ^{2} \frac{\left(a_{0}-a_{1}\right) \pi}{4}\right) .
$$

Therefore, 6.6) reduces to

$$
\frac{i c_{0}}{8} \int d x^{\prime} e^{2 x^{\prime}} \sinh \left(\sqrt{2} \beta \phi_{0}\right)\left(1-\operatorname{coth} 2\left(x^{\prime}-x_{0}\right)\right) G\left(x^{\prime}, t^{\prime} ; x^{\prime}, t^{\prime}\right),
$$

where

$$
\begin{aligned}
& G\left(x^{\prime}, t^{\prime} ; x^{\prime}, t^{\prime}\right)=\iint \frac{d \omega^{\prime}}{2 \pi} \frac{d k^{\prime}}{2 \pi} \frac{i}{\omega^{\prime 2}-k^{2}-4+i \rho}\left(f\left(k^{\prime}, x^{\prime}\right) f\left(-k^{\prime}, x^{\prime}\right)\right. \\
& +K\left(k^{\prime}\right)\left(f\left(-k^{\prime}, x^{\prime}\right)\left(f\left(-k^{\prime}, x^{\prime}\right) e^{-2 i k^{\prime} x^{\prime}}\right)\right.
\end{aligned}
$$


or after integration over $\omega^{\prime}$

$$
\begin{aligned}
G\left(x^{\prime}, t^{\prime} ; x^{\prime}, t^{\prime}\right)=\frac{1}{2} \int \frac{d k^{\prime}}{2 \pi} \frac{1}{\sqrt{k^{\prime 2}+4}}\left(f\left(k^{\prime}, x^{\prime}\right) f\left(-k^{\prime}, x^{\prime}\right)\right. \\
+K\left(k^{\prime}\right)\left(f\left(-k^{\prime}, x^{\prime}\right)\left(f\left(-k^{\prime}, x^{\prime}\right) e^{-2 i k^{\prime} x^{\prime}}\right) .\right.
\end{aligned}
$$

In fact, the above integrand has two parts, the first part can be easily manipulated but the other part which includes the exponential term is hard to calculate and we prefer to leave the computation of that part for later. Let us look at the first part of the loop propagator. The integral of this part is logarithmically divergent. Nevertheless, this divergence can be removed by an infinite renormalization of the mass parameter in the bulk potential. Then, doing the integration over $k^{\prime}$, we obtain

$$
\frac{1}{2} \int \frac{d k^{\prime}}{2 \pi} \frac{1}{\sqrt{k^{\prime 2}+4}} f\left(k^{\prime}, x^{\prime}\right) f\left(-k^{\prime}, x^{\prime}\right)=-\frac{\left(1-\operatorname{coth}^{2} 2\left(x^{\prime}-x_{0}\right)\right)}{2 \pi} .
$$

To sum up, the integral (6.9) reduces to

$$
\begin{gathered}
-\frac{i c_{0}}{16 \pi} \int_{-\infty}^{0} d x^{\prime} e^{2 x^{\prime}}\left(1-\operatorname{coth}^{2} 2\left(x^{\prime}-x_{0}\right)\right)\left(1-\operatorname{coth} 2\left(x^{\prime}-x_{0}\right)\right) \sinh \left(\sqrt{2} \beta \phi_{0}\right) \\
+\frac{i c_{0}}{16} \int_{-\infty}^{0} d x^{\prime} \int \frac{d k^{\prime}}{2 \pi} \frac{1}{\sqrt{k^{\prime 2}+4}}\left(1-\operatorname{coth} 2\left(x^{\prime}-x_{0}\right)\right) \sinh \left(\sqrt{2} \beta \phi_{0}\right) e^{2 x^{\prime}} \\
\times\left\{\frac{\left(i k^{\prime}\right)^{2}+2 i k^{\prime} \sqrt{1+\sigma_{0}} \sqrt{1+\sigma_{1}}+2\left(\sigma_{0}+\sigma_{1}\right)}{\left(i k^{\prime}\right)^{2}-2 i k^{\prime} \sqrt{1+\sigma_{0}} \sqrt{1+\sigma_{1}}+2\left(\sigma_{0}+\sigma_{1}\right)} \frac{\left(i k^{\prime}+2 \operatorname{coth} 2\left(x^{\prime}-x_{0}\right)\right)^{2}}{\left(i k^{\prime}+2\right)\left(i k^{\prime}-2\right)} e^{-2 i k^{\prime} x^{\prime}}\right\}(6
\end{gathered}
$$

The above relation has two parts and the first part which is a single integral can be performed by means of the formulae in Appendix A and we write down only the solution of this part which is expressed in terms of hypergeometric functions, that is,

$$
\begin{aligned}
\mathcal{C}_{2}=- & \frac{i c_{0}}{16 \pi} \int_{-\infty}^{0} d x^{\prime} e^{2 x^{\prime}}\left(1-\operatorname{coth}^{2} 2\left(x^{\prime}-x_{0}\right)\right)\left(1-\operatorname{coth} 2\left(x^{\prime}-x_{0}\right)\right) \sinh \left(\sqrt{2} \beta \phi_{0}\right) \\
= & \frac{i}{16 \pi}\left(1+\tan ^{2} \frac{\left(a_{0}+a_{1}\right) \pi}{4}\right)\left(1+\tan ^{2} \frac{\left(a_{0}-a_{1}\right) \pi}{4}\right) \\
\times & \left\{\frac{1}{3 \sinh 2 x_{0}}-\frac{\cosh 2 x_{0}}{24 \sinh ^{2} 2 x_{0}}-\frac{\cosh ^{2} 2 x_{0}+1}{6 \sinh ^{3} 2 x_{0}}-\frac{\cosh ^{3} 2 x_{0}+5 \cosh 2 x_{0}}{24 \sinh ^{4} 2 x_{0}}\right. \\
& -\frac{1}{6} e^{-2 x_{0}} F\left(1,1,2, e^{-4 x_{0}}\right) \\
& +\frac{11}{12} e^{-6 x_{0}} F\left(2,2,3, e^{-4 x_{0}}\right) \\
& +\frac{4}{3} e^{-10 x_{0}} F\left(3,3,4, e^{-4 x_{0}}\right) \\
& \left.+\frac{1}{2} e^{-14 x_{0}} F\left(4,4,5, e^{-4 x_{0}}\right)\right\} .
\end{aligned}
$$


So, in connection with the type III (bulk-boundary) contribution, the remaining integral is

$$
\begin{gathered}
\mathcal{C}_{3}=\frac{i c_{0}}{16} \int_{-\infty}^{0} d x^{\prime} \int \frac{d k^{\prime}}{2 \pi} \frac{1}{\sqrt{k^{\prime 2}+4}}\left(1-\operatorname{coth} 2\left(x^{\prime}-x_{0}\right)\right) \sinh \left(\sqrt{2} \beta \phi_{0}\right) e^{2 x^{\prime}} \\
\times \frac{\left(i k^{\prime}+2 \cos \frac{\left(a_{0}+a_{1}\right) \pi}{2}\right)\left(i k^{\prime}+2 \cos \frac{\left(a_{0}-a_{1}\right) \pi}{2}\right)}{\left(i k^{\prime}-2 \cos \frac{\left(a_{0}+a_{1}\right) \pi}{2}\right)\left(i k^{\prime}-2 \cos \frac{\left(a_{0}-a_{1}\right) \pi}{2}\right)} \frac{\left(i k^{\prime}+2 \operatorname{coth} 2\left(x^{\prime}-x_{0}\right)\right)^{2}}{\left(i k^{\prime}+2\right)\left(i k^{\prime}-2\right)} e^{-2 i k^{\prime} x^{\prime}} .
\end{gathered}
$$

As we mentioned before, it is more convenient to integrate over $x^{\prime}$ then afterwards over $k^{\prime}$. Since to integrate over $k^{\prime}$ first is a difficult problem. Let us do partial fraction decomposition for the rational function in (6.15). Obviously we will have four elementary partial fraction including

$$
\frac{1}{\left(i k^{\prime}-2 \cos \frac{\left(a_{0}+a_{1}\right) \pi}{2}\right)}, \frac{1}{\left(i k^{\prime}-2 \cos \frac{\left(a_{0}-a_{1}\right) \pi}{2}\right)}, \frac{1}{i k^{\prime}+2}, \frac{1}{i k^{\prime}-2} .
$$

Now, in what follows we perform the calculations in detail for one of them, for example, $\frac{1}{\left(i k^{\prime}-2 \cos \frac{\left(a_{0}+a_{1}\right) \pi}{2}\right)}$ due to the fact that for all the others the computations are similar except that $\cos \frac{\left(a_{0}+a_{1}\right) \pi}{2}$ is replaced by one of $\cos \frac{\left(a_{0}-a_{1}\right) \pi}{2},-1,1$, respectively. What we need to do is to calculate the following :

$$
\begin{aligned}
& -\frac{\left(\tan ^{2} \frac{\left(a_{0}+a_{1}\right) \pi}{4}-\cot ^{2} \frac{\left(a_{0}+a_{1}\right) \pi}{4}\right)}{\cos ^{2} \frac{\left(a_{0}+a_{1}\right) \pi}{4} \cos ^{2} \frac{\left(a_{0}-a_{1}\right) \pi}{4}} \cot \frac{a_{0} \pi}{2} \cot \frac{a_{1} \pi}{2} \\
& \times \frac{i}{16} \int_{-\infty}^{0} d x^{\prime} \int \frac{d k^{\prime}}{2 \pi} \frac{1}{\sqrt{k^{\prime 2}+4}}\left(1-\operatorname{coth} 2\left(x^{\prime}-x_{0}\right)\right) \sinh \left(\sqrt{2} \beta \phi_{0}\right) e^{\left(2-2 i k^{\prime}\right) x^{\prime}} \\
& \quad \times\left(\operatorname{coth} 2\left(x^{\prime}-x_{0}\right)+\cos \frac{\left(a_{0}+a_{1}\right) \pi}{2}\right)^{2}\left(\frac{1}{i k^{\prime}-2 \cos \frac{\left(a_{0}+a_{1}\right) \pi}{2}}\right)
\end{aligned}
$$

The integration over $x^{\prime}$ may be done by using the formulae in Appendix A and gives

$$
\begin{aligned}
& \int_{-\infty}^{0} d x^{\prime} e^{\left(2-2 i k^{\prime}\right) x^{\prime}}\left(1-\operatorname{coth} 2\left(x^{\prime}-x_{0}\right)\right) \sinh \left(\sqrt{2} \beta \phi_{0}\right) \\
& \times\left(\operatorname{coth} 2\left(x^{\prime}-x_{0}\right)+\cos \frac{\left(a_{0}+a_{1}\right) \pi}{2}\right)^{2} \\
&=\quad L\left(a_{0}, a_{1}\right) \\
&+\sum_{n=1}^{4}\left\{\frac{\left(A_{n} k^{\prime}+B_{n}\right)}{\left(k^{\prime}+2 n i\right)} e^{-(2+4(n-1)) x_{0}} F\left(n,-\frac{i}{2} k^{\prime}+n,-\frac{i}{2} k^{\prime}+n+1, e^{-4 x_{0}}\right)\right\}
\end{aligned}
$$

where

$$
L\left(a_{0}, a_{1}\right)=\left(\cos ^{2} \frac{\left(a_{0}+a_{1}\right) \pi}{2}-\frac{4}{3} \cos \frac{\left(a_{0}+a_{1}\right) \pi}{2}+\frac{2}{3}\right) \frac{1}{\sinh 2 x_{0}}
$$




$$
\begin{aligned}
& +\left(\frac{1}{2} \cos ^{2} \frac{\left(a_{0}+a_{1}\right) \pi}{2}-\cos \frac{\left(a_{0}+a_{1}\right) \pi}{2}+\frac{13}{24}\right) \frac{\cosh 2 x_{0}}{\sinh ^{2} 2 x_{0}} \\
& -\left(\frac{1}{3} \cos \frac{\left(a_{0}+a_{1}\right) \pi}{2}-\frac{1}{6}\right) \frac{\cosh ^{2} 2 x_{0}+1}{\sinh ^{3} 2 x_{0}} \\
& +\frac{1}{24} \frac{\cosh ^{3} 2 x_{0}+5 \cosh 2 x_{0}}{\sinh ^{4} 2 x_{0}}
\end{aligned}
$$

and the coefficients $A_{n}, B_{n}, n=1,2,3,4$ are constants which in fact only depend on $\cos \frac{\left(a_{0}+a_{1}\right) \pi}{2}$. Now the final calculation is to integrate over $k^{\prime}$ and it is evident that in order to do this, we have to convert the hypergeometric functions to infinite series. Considering the equation (A.9) in Appendix A, we conclude that

$$
\frac{F\left(1,-\frac{i}{2} k^{\prime}+1,-\frac{i}{2} k^{\prime}+2, e^{-4 x_{0}}\right)}{k^{\prime}+2 i}=\sum_{n=0}^{\infty} \frac{e^{-4 n x_{0}}}{k^{\prime}+i(2+2 n)} .
$$

If we differentiate both sides of the above relation with respect to $x_{0}$, then the following identity may be derived

$$
\frac{F\left(2,-\frac{i}{2} k^{\prime}+2,-\frac{i}{2} k^{\prime}+3, e^{-4 x_{0}}\right)}{k^{\prime}+4 i}=\sum_{n=1}^{\infty} \frac{n e^{-4(n-1) x_{0}}}{k^{\prime}+i(2+2 n)} .
$$

In the same way, one obtains

$$
\frac{F\left(3,-\frac{i}{2} k^{\prime}+3,-\frac{i}{2} k^{\prime}+4, e^{-4 x_{0}}\right)}{k^{\prime}+6 i}=\frac{1}{2 !} \sum_{n=2}^{\infty} \frac{n(n-1) e^{-4(n-2) x_{0}}}{k^{\prime}+i(2+2 n)}
$$

and

$$
\frac{F\left(4,-\frac{i}{2} k^{\prime}+4,-\frac{i}{2} k^{\prime}+5, e^{-4 x_{0}}\right)}{k^{\prime}+8 i}=\frac{1}{3 !} \sum_{n=3}^{\infty} \frac{n(n-1)(n-2) e^{-4(n-3) x_{0}}}{k^{\prime}+i(2+2 n)} .
$$

Now if we substitute (6.19), (6.20), (6.21) and (6.22) into (6.17), all that remains in connection with the contribution (6.16) is the integration over $k^{\prime}$. Obviously we encounter integrals of the form

$$
\int_{-\infty}^{\infty} \frac{d k^{\prime}}{\sqrt{k^{\prime 2}+4}}\left(\frac{1}{i k^{\prime}-2 \cos \frac{\left(a_{0}+a_{1}\right) \pi}{2}}\right)\left(\frac{A k^{\prime}+B}{k^{\prime}+i(2+2 n)}\right)
$$

and the $k^{\prime}$ integration may be performed by closing the contour in the upper half-plane and onto the branch cut which stretches from $k^{\prime}=2 i$ to infinity along the imaginary axis. In fact, leaving the integrals along the branch cut to be evaluated later, we obtain the required formula

$$
\int_{-\infty}^{\infty} \frac{d k^{\prime}}{\sqrt{k^{\prime 2}+4}}\left(\frac{1}{i k^{\prime}-2 \cos \frac{\left(a_{0}+a_{1}\right) \pi}{2}}\right)\left(\frac{A k^{\prime}+B}{k^{\prime}+i(2+2 n)}\right)
$$




$$
\begin{aligned}
= & \frac{\left(2 \cos \frac{\left(a_{0}+a_{1}\right) \pi}{2} A+i B\right)}{\left(2 n+2-2 \cos \frac{\left(a_{0}+a_{1}\right) \pi}{2}\right)} \frac{\frac{\left(a_{0}+a_{1}\right) \pi}{2}}{\sin \frac{\left(a_{0}+a_{1}\right) \pi}{2}} \\
& -\frac{((2 n+2) A+i B)}{\left(2 n+2-2 \cos \frac{\left(a_{0}+a_{1}\right) \pi}{2}\right)} \frac{1}{2 \sqrt{n^{2}+2 n}} \ln \left\{\frac{n+1-\sqrt{n^{2}+2 n}}{n+1+\sqrt{n^{2}+2 n}}\right\} .
\end{aligned}
$$

Note $(6.24)$ is valid when $n \neq 0$, on the other hand if $n=0$ then one may find

$$
\begin{aligned}
& \int_{-\infty}^{\infty} \frac{d k^{\prime}}{\sqrt{k^{\prime 2}+4}}\left(\frac{1}{i k^{\prime}-2 \cos \frac{\left(a_{0}+a_{1}\right) \pi}{2}}\right)\left(\frac{A k^{\prime}+B}{k^{\prime}+2 i}\right) \\
& =\frac{\left(2 \cos \frac{\left(a_{0}+a_{1}\right) \pi}{2} A+i B\right)}{\left(2-2 \cos \frac{\left(a_{0}+a_{1}\right) \pi}{2}\right)} \frac{\frac{\left(a_{0}+a_{1}\right) \pi}{2}}{\sin \frac{\left(a_{0}+a_{1}\right) \pi}{2}}-\frac{(2 A+i B)}{\left(2-2 \cos \frac{\left(a_{0}+a_{1}\right) \pi}{2}\right)} .
\end{aligned}
$$

Now we are in a position to write down (6.16) or, in fact, the integral (6.15)

$$
\begin{aligned}
& \mathcal{C}_{3}=\frac{i}{32 \pi} \frac{\left(\tan ^{2} \frac{\left(a_{0}+a_{1}\right) \pi}{4}-\cot ^{2} \frac{\left(a_{0}+a_{1}\right) \pi}{4}\right)}{\cos ^{2} \frac{\left(a_{0}+a_{1}\right) \pi}{4} \cos ^{2} \frac{\left(a_{0}-a_{1}\right) \pi}{4}} \cot \frac{a_{0} \pi}{2} \cot \frac{a_{1} \pi}{2} \\
& \times\left\{\frac{\frac{\left(a_{0}+a_{1}\right) \pi}{2}}{\sin \frac{\left(a_{0}+a_{1}\right) \pi}{2}} L\left(a_{0}, a_{1}\right)\right. \\
& +\frac{1}{12} e^{-2 x_{0}}\left(\frac{2 A_{1}+i B_{1}}{2-2 \cos \frac{\left(a_{0}+a_{1}\right) \pi}{2}}-\frac{2 \cos \frac{\left(a_{0}+a_{1}\right) \pi}{2} A_{1}+i B_{1}}{2-2 \cos \frac{\left(a_{0}+a_{1}\right) \pi}{2}} \frac{\frac{\left(a_{0}+a_{1}\right) \pi}{2}}{\sin \frac{\left(a_{0}+a_{1}\right) \pi}{2}}\right) \\
& -\sum_{n=1}^{\infty} \frac{\frac{\left(a_{0}+a_{1}\right) \pi}{2}}{\sin \frac{\left(a_{0}+a_{1}\right) \pi}{2}} \frac{e^{-(2+4 n) x_{0}}}{\left(2 n+2-2 \cos \frac{\left(a_{0}+a_{1}\right) \pi}{2}\right)}\left[\left(2 \cos \frac{\left(a_{0}+a_{1}\right) \pi}{2} A_{1}+i B_{1}\right)\right. \\
& +\frac{n}{1 !}\left(2 \cos \frac{\left(a_{0}+a_{1}\right) \pi}{2} A_{2}+i B_{2}\right)+\frac{n(n-1)}{2 !}\left(2 \cos \frac{\left(a_{0}+a_{1}\right) \pi}{2} A_{3}+i B_{3}\right) \\
& \left.+\frac{n(n-1)(n-2)}{3 !}\left(2 \cos \frac{\left(a_{0}+a_{1}\right) \pi}{2} A_{4}+i B_{4}\right)\right] \\
& +\sum_{n=1}^{\infty} \frac{e^{-(2+4 n) x_{0}}}{\left(2 n+2-2 \cos \frac{\left(a_{0}+a_{1}\right) \pi}{2}\right)} \frac{1}{2 \sqrt{n^{2}+2 n}} \ln \left\{\frac{n+1-\sqrt{n^{2}+2 n}}{n+1+\sqrt{n^{2}+2 n}}\right\} \\
& \left(\left[(2 n+2) A_{1}+i B_{1}\right]+\frac{n}{1 !}\left[(2 n+2) A_{2}+i B_{2}\right]\right. \\
& \left.\left.+\frac{n(n-1)}{2 !}\left[(2 n+2) A_{3}+i B_{3}\right]+\frac{n(n-1)(n-2)}{3 !}\left[(2 n+2) A_{4}+i B_{4}\right]\right)\right\}
\end{aligned}
$$

+ other pole pieces.

Note, in the above expression all the series are convergent. As we mentioned before, (6.26) must be considered (after adding to (6.14)) as a coefficient factor of (6.4) in order to constitute the type III (bulk-boundary) contribution i.e.:

$$
\mathcal{C}=\mathcal{C}_{1}\left(\mathcal{C}_{2}+\mathcal{C}_{3}\right) \text {. }
$$




\section{Type I diagram}

In this section we calculate the contribution of the type I Feynman diagram to the classical reflection factor when the vertex is placed inside the bulk region. In fact, when the vertex is located at the boundary then, the corresponding contribution has been found 24] and is given by

$$
\begin{aligned}
& -\frac{i \beta^{2}}{8}\left(\sigma_{1} \operatorname{coth} x_{0}+\sigma_{0} \tanh x_{0}\right)\left(a_{0} \cot a_{0} \pi+a_{1} \cot a_{1} \pi\right) \\
& \times \int \frac{d \omega}{2 \pi} e^{-i \omega\left(t_{1}-t_{2}\right)} e^{-i \hat{k}\left(x_{1}+x_{2}\right)} \frac{i \hat{k}+2 \operatorname{coth} 2\left(x_{1}-x_{0}\right)}{P(\hat{k})} \frac{i \hat{k}+2 \operatorname{coth} 2\left(x_{2}-x_{0}\right)}{P(\hat{k})}(7.1)
\end{aligned}
$$

Clearly, in our case the bulk four point coupling should be considered in the interaction vertex. Moreover, as before, the combinatorial factor associated with this diagram will appear as a coefficient. Hence, the contribution has the form

$$
-4 i \beta^{2} \int_{-\infty}^{\infty} d t \int_{-\infty}^{0} d x G\left(x_{1}, t_{1} ; x, t\right) G(x, t ; x, t) G\left(x, t ; x_{2}, t_{2}\right) \cosh \left(\sqrt{2} \beta \phi_{0}\right)
$$

where

$$
\cosh \left(\sqrt{2} \beta \phi_{0}\right)=\left(2 \operatorname{coth}^{2} 2\left(x-x_{0}\right)-1\right) .
$$

In the previous section, we simplified the loop propagator to

$$
\begin{aligned}
G(x, t ; x, t)= & -\frac{\left(1-\operatorname{coth}^{2} 2\left(x-x_{0}\right)\right)}{2 \pi} \\
& +\frac{1}{2} \int \frac{d k^{\prime \prime}}{2 \pi} \frac{1}{\sqrt{k^{\prime \prime 2}+4}} K\left(k^{\prime \prime}\right) f\left(-k^{\prime \prime}, x\right) f\left(-k^{\prime \prime}, x\right) e^{-2 i k^{\prime \prime} x} .
\end{aligned}
$$

Also the integral part of the loop Green function is hard enough to evaluate and we found out that it is better to do this integration during the final stage. Now, let us rewrite the contribution $(\overline{7.2})$ in the expanded form

$$
\begin{aligned}
& -4 i \beta^{2} \int d t \int_{-\infty}^{0} d x \iint \frac{d \omega}{2 \pi} \frac{d k}{2 \pi} e^{-i \omega\left(t_{1}-t\right)} \frac{i}{\omega^{2}-k^{2}-4+i \rho}\left(f\left(k, x_{1}\right) f(-k, x) e^{i k\left(x_{1}-x\right)}\right. \\
& \left.+K(k) f\left(-k, x_{1}\right) f(-k, x) e^{-i k\left(x_{1}+x\right)}\right) \cosh \left(\sqrt{2} \beta \phi_{0}\right) \\
& \times\left\{-\frac{\left(1-\operatorname{coth}^{2} 2\left(x-x_{0}\right)\right)}{2 \pi}+\frac{1}{2} \int \frac{d k^{\prime \prime}}{2 \pi} \frac{1}{\sqrt{k^{\prime \prime 2}+4}} K\left(k^{\prime \prime}\right) f\left(-k^{\prime \prime}, x\right) f\left(-k^{\prime \prime}, x\right) e^{-2 i k^{\prime \prime} x}\right\} \\
& \times \iint \frac{d \omega^{\prime}}{2 \pi} \frac{d k^{\prime}}{2 \pi} e^{-i \omega^{\prime}\left(t-t_{2}\right)} \frac{i}{\omega^{\prime 2}-k^{\prime 2}-4+i \rho}\left(f\left(k^{\prime}, x\right) f\left(-k^{\prime}, x_{2}\right) e^{i k^{\prime}\left(x-x_{2}\right)}\right. \\
& \left.+K^{\prime}\left(k^{\prime}\right) f\left(-k^{\prime}, x\right) f\left(-k^{\prime}, x_{2}\right) e^{-i k^{\prime}\left(x+x_{2}\right)}\right) .
\end{aligned}
$$

Looking at (7.5), one can predict that the calculations will be lengthy and intri-

cate. The starting point is to do the $t$ integration which allows the substitution $\omega=\omega^{\prime}$. 
Secondly, it is necessary to perform a transformation $k \rightarrow-k$ in the first term of the first propagator. Moreover, if we multiply the first and the third propagator with each other, then obviously we will have four pole pieces and fortunately if we do the calculation for one of them (for example the first one), then the calculations corresponding to the other three pole pieces may be treated similarly with $k+k^{\prime}$ replaced by one of $k-k^{\prime},-k+k^{\prime}$ and $-k-k^{\prime}$. Because of this in what follows we follow the problem only for one pole piece. Hence our problem is, in fact, the following integral

$$
\begin{aligned}
\mathcal{D}=-4 i \beta^{2} \int_{-\infty}^{0} d x \iint \frac{d \omega}{2 \pi} \frac{d k}{2 \pi} e^{-i \omega\left(t_{1}-t_{2}\right)} \frac{i}{\omega^{2}-k^{2}-4+i \rho} \\
\times \cosh \left(\sqrt{2} \beta \phi_{0}\right) f\left(-k, x_{1}\right) f(k, x) e^{-i k\left(x_{1}-x\right)} \\
\times\left(-\frac{\left(1-\operatorname{coth}^{2} 2\left(x-x_{0}\right)\right)}{2 \pi}+\frac{1}{2} \int \frac{d k^{\prime \prime}}{2 \pi} \frac{1}{\sqrt{k^{\prime \prime 2}+4}} K\left(k^{\prime \prime}\right) f\left(-k^{\prime \prime}, x\right) f\left(-k^{\prime \prime}, x\right) e^{-2 i k^{\prime \prime} x}\right) \\
\times \int \frac{d k^{\prime}}{2 \pi} \frac{i}{\omega^{2}-k^{\prime 2}-4+i \rho} f\left(k^{\prime}, x\right) f\left(-k^{\prime}, x_{2}\right) e^{i k^{\prime}\left(x-x_{2}\right)} .
\end{aligned}
$$

In fact, the above contribution has two parts. The first part, in which the integral of the middle momentum $\left(k^{\prime \prime}\right)$ is not involved, can be calculated by means of the formulae in Appendix B and we call this part $\mathcal{D}_{1}$. Let us write down the solution of this part. This contribution is expressed in terms of the hypergeometric function as :

$$
\begin{aligned}
\mathcal{D}_{1}=\frac{i \beta^{2}}{\pi} \int \frac{d \omega}{2 \pi} e^{-i \omega\left(t_{1}-t_{2}\right)} e^{-i \hat{k}\left(x_{1}+x_{2}\right)} f\left(-\hat{k}, x_{1}\right) f\left(-\hat{k}, x_{2}\right) \frac{1}{\hat{k}^{2}} \\
\times\left\{\frac{4 i-4 \hat{k}-i \hat{k}^{2}}{\hat{k}-2 i} e^{-4 x_{0}} F\left(2, \frac{i}{2} \hat{k}+1, \frac{i}{2} \hat{k}+2, e^{-4 x_{0}}\right)\right. \\
+\frac{48 i-40 \hat{k}-8 i \hat{k}^{2}}{\hat{k}-4 i} e^{-8 x_{0}} F\left(3, \frac{i}{2} \hat{k}+2, \frac{i}{2} \hat{k}+3, e^{-4 x_{0}}\right) \\
+\frac{176 i-96 \hat{k}-8 i \hat{k}^{2}}{\hat{k}-6 i} e^{-12 x_{0}} F\left(4, \frac{i}{2} \hat{k}+3, \frac{i}{2} \hat{k}+4, e^{-4 x_{0}}\right) \\
+\frac{256 i-64 \hat{k}}{\hat{k}-8 i} e^{-16 x_{0}} F\left(5, \frac{i}{2} \hat{k}+4, \frac{i}{2} \hat{k}+5, e^{-4 x_{0}}\right) \\
\left.+\frac{128 i}{\hat{k}-10 i} e^{-20 x_{0}} F\left(6, \frac{i}{2} \hat{k}+5, \frac{i}{2} \hat{k}+6, e^{-4 x_{0}}\right)\right\}
\end{aligned}
$$

Now it is better for the second part, which we call $\mathcal{D}_{2}$, to integrate first over $x$ then over $k^{\prime \prime}$. Meanwhile, before starting the integration, it is useful to note that if we do the partial fraction decomposition for $K^{\prime \prime}\left(k^{\prime \prime}\right) f\left(-k^{\prime \prime}, x\right) f\left(-k^{\prime \prime}, x\right)$, then we will have four elementary partial fractions as

$$
\frac{1}{\left(i k^{\prime \prime}-2 \cos \frac{\left(a_{0}+a_{1}\right) \pi}{2}\right)}, \frac{1}{\left(i k^{\prime \prime}-2 \cos \frac{\left(a_{0}-a_{1}\right) \pi}{2}\right)}, \frac{1}{i k^{\prime \prime}+2}, \frac{1}{i k^{\prime \prime}-2} \text {. }
$$


As before, in the remaining section we continue the computations in detail for one of them (for example, $\frac{1}{\left(i k^{\prime \prime}-2 \cos \frac{\left(a_{0}+a_{1}\right) \pi}{2}\right)}$ ) because the calculations corresponding to the other three elementary partial fractions can be done in the same manner just by the substitution of $\cos \frac{\left(a_{0}+a_{1}\right) \pi}{2}$ by one of $\cos \frac{\left(a_{0}-a_{1}\right) \pi}{2},-1,1$, respectively. So, our problem reduces to this integral

$$
\begin{aligned}
& 2 i \beta^{2} \cot \frac{a_{0} \pi}{2} \cot \frac{a_{1} \pi}{2}\left(\tan ^{2} \frac{\left(a_{0}+a_{1}\right) \pi}{4}-\cot ^{2} \frac{\left(a_{0}+a_{1}\right) \pi}{4}\right) \\
& \times \int_{-\infty}^{0} d x \iint \frac{d \omega}{2 \pi} \frac{d k}{2 \pi} e^{-i \omega\left(t_{1}-t_{2}\right)} \frac{i}{\omega^{2}-k^{2}-4+i \rho} \\
& \times \cosh \left(\sqrt{2} \beta \phi_{0}\right) f\left(-k, x_{1}\right) f(k, x) e^{i k\left(x_{1}-x\right)} \\
& \times \int \frac{d k^{\prime \prime}}{2 \pi} \frac{1}{\sqrt{k^{\prime \prime 2}+4}} \frac{e^{-2 i k^{\prime \prime} x}}{i k^{\prime \prime}-2 \cos \frac{\left(a_{0}+a_{1}\right) \pi}{2}}\left(\operatorname{coth} 2\left(x-x_{0}\right)+\cos \frac{\left(a_{0}+a_{1}\right) \pi}{2}\right)^{2} \\
& \times \int \frac{d k^{\prime}}{2 \pi} \frac{i}{\omega^{2}-k^{\prime 2}-4+i \rho} f\left(k^{\prime}, x\right) f\left(-k^{\prime}, x_{2}\right) e^{i k^{\prime}\left(x-x_{2}\right)} .
\end{aligned}
$$

The integration over $x$ can be evaluated by means of the formulae given in Appendix B as

$$
\begin{aligned}
& \int_{-\infty}^{0} d x e^{i\left(k+k^{\prime}-2 k^{\prime \prime}\right) x} \cosh \left(\sqrt{2} \beta \phi_{0}\right) f(k, x) f\left(k^{\prime}, x\right)\left(\operatorname{coth} 2\left(x-x_{0}\right)+\cos \frac{\left(a_{0}+a_{1}\right) \pi}{2}\right)^{2} \\
& =\frac{1}{(i k+2)\left(i k^{\prime}+2\right)} \frac{\left(A_{0}^{\prime} k k^{\prime}+B_{0}^{\prime}\left(k+k^{\prime}\right)+C_{0}^{\prime}\right)}{\left(k+k^{\prime}-2 k^{\prime \prime}\right)} \\
& +\frac{1}{(i k+2)\left(i k^{\prime}+2\right)} \sum_{n=1}^{6}\left\{\frac{\left(A_{n}^{\prime} k k^{\prime}+B_{n}^{\prime}\left(k+k^{\prime}\right)+C_{n}^{\prime}\right)}{\left(k+k^{\prime}-2 k^{\prime \prime}-4(n-1) i\right)} e^{-4(n-1) x_{0}}\right. \\
& \left.\quad \times F\left(n, \frac{i}{4}\left(k+k^{\prime}-2 k^{\prime \prime}\right)+n-1, \frac{i}{4}\left(k+k^{\prime}-2 k^{\prime \prime}\right)+n, e^{-4 x_{0}}\right)\right\}
\end{aligned}
$$

where the coefficients $A_{n}^{\prime}, B_{n}^{\prime}, C_{n}^{\prime} ; n=0,1, . ., 6$ are constants and depend only on $\cos \frac{\left(a_{0}+a_{1}\right) \pi}{2}$. In fact $A_{5}^{\prime}, A_{6}^{\prime}$ and $B_{6}^{\prime}$ are zero. Now the subsequent calculation is to integrate over $k^{\prime \prime}$ and it is clear that to do this, it is necessary to convert the hypergeometric function to an infinite series. Looking at (B.9), we may write down

$$
\frac{F\left(1, \frac{i}{4}\left(k+k^{\prime}-2 k^{\prime \prime}\right), \frac{i}{4}\left(k+k^{\prime}-2 k^{\prime \prime}\right)+1, e^{-4 x_{0}}\right)}{k+k^{\prime}-2 k^{\prime \prime}}=\sum_{n=0}^{\infty} \frac{e^{-4 n x_{0}}}{k+k^{\prime}-2 k^{\prime \prime}-4 n i}
$$

and by differentiating both sides of the above relation with respect to $x_{0}$, then we obtain

$$
\frac{F\left(2, \frac{i}{4}\left(k+k^{\prime}-2 k^{\prime \prime}\right)+1, \frac{i}{4}\left(k+k^{\prime}-2 k^{\prime \prime}\right)+2, e^{-4 x_{0}}\right)}{k+k^{\prime}-2 k^{\prime \prime}-4 i}=\sum_{n=1}^{\infty} \frac{n e^{-4(n-1) x_{0}}}{k+k^{\prime}-2 k^{\prime \prime}-4 n i} .
$$


Similarly one can derive the infinite series forms of the other hypergeometric functions as

$$
\begin{gathered}
\frac{F\left(3, \frac{i}{4}\left(k+k^{\prime}-2 k^{\prime \prime}\right)+2, \frac{i}{4}\left(k+k^{\prime}-2 k^{\prime \prime}\right)+3, e^{-4 x_{0}}\right)}{k+k^{\prime}-2 k^{\prime \prime}-8 i}=\frac{1}{2 !} \sum_{n=2}^{\infty} \frac{n(n-1) e^{-4(n-2) x_{0}}}{k+k^{\prime}-2 k^{\prime \prime}-4 n i}, \\
\frac{F\left(4, \frac{i}{4}\left(k+k^{\prime}-2 k^{\prime \prime}\right)+3, \frac{i}{4}\left(k+k^{\prime}-2 k^{\prime \prime}\right)+4, e^{-4 x_{0}}\right)}{k+k^{\prime}-2 k^{\prime \prime}-12 i} \\
=\frac{1}{3 !} \sum_{n=3}^{\infty} \frac{n(n-1)(n-2) e^{-4(n-3) x_{0}}}{k+k^{\prime}-2 k^{\prime \prime}-4 n i}, \\
\frac{F\left(5, \frac{i}{4}\left(k+k^{\prime}-2 k^{\prime \prime}\right)+4, \frac{i}{4}\left(k+k^{\prime}-2 k^{\prime \prime}\right)+5, e^{-4 x_{0}}\right)}{k+k^{\prime}-2 k^{\prime \prime}-16 i} \\
=\frac{1}{4 !} \sum_{n=4}^{\infty} \frac{n(n-1)(n-2)(n-3) e^{-4(n-4) x_{0}}}{k+k^{\prime}-2 k^{\prime \prime}-4 n i}
\end{gathered}
$$

and

$$
\begin{aligned}
\frac{F\left(6, \frac{i}{4}\left(k+k^{\prime}-\right.\right.}{\left.\left.2 k^{\prime \prime}\right)+5, \frac{i}{4}\left(k+k^{\prime}-2 k^{\prime \prime}\right)+6, e^{-4 x_{0}}\right)} \\
k+k^{\prime}-2 k^{\prime \prime}-20 i \\
=\frac{1}{5 !} \sum_{n=5}^{\infty} \frac{n(n-1)(n-2)(n-3)(n-4) e^{-4(n-5) x_{0}}}{k+k^{\prime}-2 k^{\prime \prime}-4 n i}
\end{aligned}
$$

Let us substitute (7.10), (7.11), (7.12), (7.13), (7.14) and (7.15) in (7.9) and obviously what remains in connection with the contribution (7.8), are the integrations over $k^{\prime \prime}, k^{\prime}$ and $k$. As before in previous sections, in order to integrate over the momenta $k$ and $k^{\prime}$, it is sufficient to close the contours in the upper half-plane and pick up poles at $\hat{k}=k=k^{\prime}=\sqrt{\omega^{2}-4}$ as all the other poles' contributions will be exponentially damped when $x, x^{\prime} \rightarrow-\infty$. Meanwhile, the integration over $k^{\prime \prime}$ is of the form

$$
\int \frac{d k^{\prime \prime}}{\sqrt{k^{\prime \prime 2}+4}} \frac{1}{\left(i k^{\prime \prime}-2 \cos \frac{\left(a_{0}+a_{1}\right) \pi}{2}\right)} \frac{1}{\left(k+k^{\prime}-2 k^{\prime \prime}-4 n i\right)} .
$$

Now to manipulate the integral, let us choose the contour in the upper half-plane, taking care of the branch cut which runs from $k^{\prime \prime}=2 i$ to infinity along the imaginary axis. Clearly this integral reduces to the integrals along the branch cut and we obtain

$$
\begin{aligned}
& \int \frac{d k^{\prime \prime}}{\sqrt{k^{\prime \prime 2}+4}} \frac{1}{\left(i k^{\prime \prime}-2 \cos \frac{\left(a_{0}+a_{1}\right) \pi}{2}\right)} \frac{1}{\left(k+k^{\prime}-2 k^{\prime \prime}-4 n i\right)} \\
& =\frac{1}{\left(k+k^{\prime}+4 i \cos \frac{\left(a_{0}+a_{1}\right) \pi}{2}-4 n i\right)}\left(-\frac{\frac{\left(a_{0}+a_{1}\right) \pi}{2}}{\sin \frac{\left(a_{0}+a_{1}\right) \pi}{2}}\right.
\end{aligned}
$$




$$
\begin{aligned}
& -\frac{2 i}{\sqrt{\frac{\left(k+k^{\prime}\right)^{2}}{4}+4-4 n^{2}-2 n i\left(k+k^{\prime}\right)}} \\
& \left.\times \ln \left\{\frac{1+\frac{i\left(k+k^{\prime}\right)}{4}+n+\frac{i}{2} \sqrt{\frac{\left(k+k^{\prime}\right)^{2}}{4}+4-4 n^{2}-2 n i\left(k+k^{\prime}\right)}}{1+\frac{i\left(k+k^{\prime}\right)}{4}+n-\frac{i}{2} \sqrt{\frac{\left(k+k^{\prime}\right)^{2}}{4}+4-4 n^{2}-2 n i\left(k+k^{\prime}\right)}}\right\}\right) .
\end{aligned}
$$

When $n=0$, the above formula is simplified much more, especially after doing the integration over $k$ and $k^{\prime}$ and using the fact that $\hat{k}=k=k^{\prime}=2 \sinh \theta$. So the following formula can be obtained

$$
\begin{aligned}
& \int \frac{d k^{\prime \prime}}{\sqrt{k^{\prime 2}+4}} \frac{1}{\left(i k^{\prime \prime}-2 \cos \frac{\left(a_{0}+a_{1}\right) \pi}{2}\right)} \frac{1}{\left(2 \hat{k}-2 k^{\prime \prime}\right)} \\
& =\frac{1}{\left(2 \hat{k}+4 i \cos \frac{\left(a_{0}+a_{1}\right) \pi}{2}\right)}\left(-\frac{\frac{\left(a_{0}+a_{1}\right) \pi}{2}}{\sin \frac{\left(a_{0}+a_{1}\right) \pi}{2}}+\frac{2}{\sqrt{\hat{k}^{2}+4}}\left(\frac{\pi}{2}-i \theta\right)\right) .
\end{aligned}
$$

Now, (7.8) or more generally the contribution $\mathcal{D}_{2}$ can be written as:

$$
\begin{aligned}
\mathcal{D}_{2}= & \frac{i \beta^{2}}{4 \pi} \cot \frac{a_{0} \pi}{2} \cot \frac{a_{1} \pi}{2}\left(\tan ^{2} \frac{\left(a_{0}+a_{1}\right) \pi}{4}-\cot ^{2} \frac{\left(a_{0}+a_{1}\right) \pi}{4}\right) \\
& \times \int \frac{d \omega}{2 \pi} e^{-i \omega\left(t_{1}-t_{2}\right)} e^{-i \hat{k}\left(x_{1}+x_{2}\right)}\left(\frac{1}{\hat{k}}\right)^{2} f\left(-\hat{k}, x_{1}\right) f\left(-\hat{k}, x_{2}\right) \\
& \times\left\{\frac{2 i\left(1-\cos \frac{\left(a_{0}+a_{1}\right) \pi}{2}\right)^{2}}{\left(\hat{k}+2 i \cos \frac{\left(a_{0}+a_{1}\right) \pi}{2}\right)}\left(\frac{\frac{\left(a_{0}+a_{1}\right) \pi}{2}}{\sin \frac{\left(a_{0}+a_{1}\right) \pi}{2}}-\frac{2}{\sqrt{\hat{k}^{2}+4}}\left(\frac{\pi}{2}-i \theta\right)\right)\right. \\
& -\frac{1}{(i \hat{k}+2)^{2}} \sum_{n=1}^{\infty} \frac{e^{-4 n x_{0}}}{\left(2 \hat{k}+4 i \cos \frac{\left(a_{0}+a_{1}\right) \pi}{2}-4 n i\right)}\left(\frac{\frac{\left(a_{0}+a_{1}\right) \pi}{2}}{\sin \frac{\left(a_{0}+a_{1}\right) \pi}{2}}\right. \\
& \left.+\frac{2 i}{\sqrt{\hat{k}^{2}+4-4 n^{2}-4 n i \hat{k}}} \ln \left\{\frac{1+\frac{i}{2} \hat{k}+n+\frac{i}{2} \sqrt{\hat{k}^{2}+4-4 n^{2}-4 n i \hat{k}}}{1+\frac{i}{2} \hat{k}+n-\frac{i}{2} \sqrt{\hat{k}^{2}+4-4 n^{2}-4 n i \hat{k}}}\right\}\right) \\
& +\frac{n(n-1)(n-2)}{3 !}\left(A_{1}^{\prime} \hat{k}^{2}+2 B_{1}^{\prime} \hat{k}+2 B_{4}^{\prime} \hat{k}+C_{4}^{\prime}\right)+n\left(A_{2}^{\prime} \hat{k}^{2}+2 B_{2}^{\prime} \hat{k}+C_{2}^{\prime}\right)+\frac{n(n-1)}{2 !}\left(A_{3}^{\prime} \hat{k}^{2}+2 B_{3}^{\prime} \hat{k}+C_{3}^{\prime}\right) \\
& \left.\left.+\frac{n(n-1)(n-2)(n-3)(n-4)}{5 !} C_{6}^{\prime}\right)\right\} \\
+ & \text { other pole pieces. }
\end{aligned}
$$

Firstly, in order to check the above solution, if we set $a_{0}=a_{1}$ and consider the other pole pieces then, we can derive the formula (3.10) in reference [14. As we mentioned before, the calculation of this reference is based on the case when the boundary parameters are equal. Secondly, in this solution, we verified that the term which depends explicitly on 
the rapidity of the particle $(\theta)$ is cancelled by counterpart terms in the other pole pieces. It is evident that if we add the expressions (7.7) and (7.19) then, the contribution (7.6) will be obtained i.e. $\mathcal{D}=\mathcal{D}_{1}+\mathcal{D}_{2}$.

\section{Discussion}

Affine Toda field theory on the whole line is an exactly solvable theory for which the S-matrices have been formulated. However, when a boundary is present then the boundary S-matrices of the theory i.e. the reflection factors, have not been completely found. The bootstrap technique does not uniquely determine the reflection factors. Fortunately perturbation theory provides the link between the expressions for the reflection factors which come from the bootstrap equations and the boundary parameters. Nevertheless, this method normally involves complicated calculations.

In this paper the quantum reflection factor for the $a_{1}^{(1)}$ affine Toda field theory or sinh-Gordon model with integrable boundary conditions has been studied in low order perturbation theory when $\sigma_{0} \neq \sigma_{1}$. It is found that at one loop order the quantum corrections to the classical reflection factor of the model can be expressed in terms of hypergeometric functions for most of the related Feynman diagrams. Although there is still some work to do to calculate the contributions of the remaining diagrams, it is understood that the provided procedure and some formalisms may be followed for them.

The calculations corresponding to the type II Feynman diagram which are not carried out in this paper, are more difficult than the others. In this case the two middle propagators are exactly the same and this fact influences the difficulty of the computations. However some formulae that have been presented here, could be helpful for the remaining diagram. For example, consider the contribution of the type II (boundary-bulk) diagram:

$$
\begin{aligned}
-2 \beta^{2}\left(\sigma_{1} \operatorname{coth} x_{0}-\sigma_{0} \tanh x_{0}\right) \int & \iint d t d t^{\prime} d x G\left(x_{1}, t_{1} ; x, t\right) G\left(x, t ; 0, t^{\prime}\right) \\
& \times G\left(x, t ; 0, t^{\prime}\right) G\left(0, t^{\prime} ; x_{2}, t_{2}\right) \sinh \left(\sqrt{2} \beta \phi_{0}\right) .
\end{aligned}
$$

Now as far as the integration over $x$ is concerned we should obtain the following integrals

$$
\int_{-\infty}^{0} d x e^{i\left(k+k^{\prime}-k_{1}\right) x} \sinh \left(\sqrt{2} \beta \phi_{0}\right) \operatorname{coth}^{n} 2\left(x-x_{0}\right)
$$

where $\mathrm{n}=0,1,2,3$. It is better to solve:

$$
\int_{-\infty}^{0} d x \exp \left\{\tau+i\left(k+k^{\prime}-k_{1}\right) x\right\} \sinh \left(\sqrt{2} \beta \phi_{0}\right) \operatorname{coth}^{n} 2\left(x-x_{0}\right)
$$

in which $\tau$ is a small positive quantity and will be taken to zero later. In fact, the relation (8.3) is very similar to the formula (A.1) in Appendix A. So, following the 
same procedure that have been followed in Appendix A, one can find the solution of (8.2) when $n=0$ as:

$$
\begin{aligned}
& \int_{-\infty}^{0} d x \quad e^{i\left(k+k^{\prime}-k_{1}\right) x} \sinh \left(\sqrt{2} \beta \phi_{0}\right) \\
&= \frac{1}{\sinh 2 x_{0}} \\
&-\frac{2\left(k+k^{\prime}-k_{1}\right)}{k+k^{\prime}-k_{1}-2 i} \\
& \times e^{-2 x_{0}} F\left(1, \frac{i}{4}\left(k+k^{\prime}-k_{1}\right)+\frac{1}{2}, \frac{i}{4}\left(k+k^{\prime}-k_{1}\right)+\frac{3}{2}, e^{-4 x_{0}}\right) .
\end{aligned}
$$

Then, the solutions of (8.2) for $n=1,2,3$ can be derived exactly in according to the Appendix A terms. But, this is not all of the problem. As we mentioned before, in type II diagram double Green functions cause the middle momenta to be linked to each other in a complicated way and the calculations become more intricate. Actually this diagram must be studied in three cases depending on the interaction vertices being located in the bulk region or at the boundary. Moreover because of the symmetry, the contribution of the type II (boundary-bulk) diagram is the same as the type II (bulk-boundary) one.

When the boundary parameters are equal then, only the type I diagram is involved in the theory. As we mentioned before, in this special case [14] the quantum corrections to the classical reflection of the model have been found and Ghoshal's formula for the lightest breather is checked perturbatively to $O\left(\beta^{2}\right)$. In our case, we realised that the contribution of the type I (bulk) reduces to the special case. Taking (7.7) and (7.19) expressions, if we put $\sigma_{0}=\sigma_{1}$ then, we obtain the same result as reference [14 and this is a check on our calculations. Moreover, when $\sigma_{0} \neq \sigma_{1}$ the following expressions for $E$ and $F$ in Ghoshal's formula (2.8) have been conjectured [21] to be:

$$
E=\left(a_{0}+a_{1}\right)(1-B / 2) \quad F=\left(a_{0}-a_{1}\right)(1-B / 2) .
$$

So, it will be interesting to check the above conjecture after finding the contributions of the remaining diagrams and adding the results all together. This will lead to a deeper understanding of the quantum integrability of the theory. However, it is necessary to find simplifications of the contributions when they add among themselves in order to get Ghoshal's formula.

\section{Acknowledgement}

We would like to thank E. Corrigan and P. Bowcock for encouragement, discussions and suggestions, and the Ministry of Culture and Higher Education of Iran for financial support. 


\section{Appendix A}

In this Appendix we obtain such integrals

$$
S_{n}=\int_{-\infty}^{0} d x e^{(2+i k) x} \sinh \left(\sqrt{2} \beta \phi_{0}\right) \operatorname{coth}^{n} 2\left(x-x_{0}\right)
$$

in which $n=0,1,2,3, \phi_{0}$ is the background solution to the equation of field so that $\sinh \left(\sqrt{2} \beta \phi_{0}\right)$ is proportional to the bulk three point coupling which is given by

$$
\sinh \left(\sqrt{2} \beta \phi_{0}\right)=2 \cosh 2\left(x-x_{0}\right)\left(\operatorname{coth}^{2} 2\left(x-x_{0}\right)-1\right) .
$$

Let us start with the simplest case when $n=0$. Using (A.2), we have

$$
S_{0}=-\int_{-\infty}^{0} e^{(2+i k) x} d\left(\frac{1}{\sinh 2\left(x-x_{0}\right)}\right)
$$

or, after integration by parts

$$
S_{0}=\frac{1}{\sinh 2 x_{0}}+2(2+i k) \int_{-\infty}^{0} d x e^{(2+i k) x} \frac{1}{e^{2\left(x-x_{0}\right)}-e^{-2\left(x-x_{0}\right)}} .
$$

Now, according to (3.6), if $\sigma_{0}>\sigma_{1}$ then $x_{0} \geq 0$. Otherwise, it is necessary to adjust the background solution (3.5) by shifting $x_{0}$ through $i \pi / 2$ in order to be guaranteed that $x_{0} \geq 0$. The singularity in the equation (3.5) is unimportant provided $x_{0}$ is positive. So, from now on it is assumed $\sigma_{0} \geq \sigma_{1}$. Therefore, $x_{0}$ is greater or equal to zero. But, $x$ is less than zero, so $0<e^{4\left(x-x_{0}\right)}<1$ and hence

$$
\frac{1}{e^{2\left(x-x_{0}\right)}-e^{-2\left(x-x_{0}\right)}}=-e^{2\left(x-x_{0}\right)} \sum_{n=0}^{\infty} e^{4 n\left(x-x_{0}\right)} .
$$

Substituting (A.5) in (A.4), we obtain

$$
S_{0}=\frac{1}{\sinh 2 x_{0}}-2(2+i k) e^{-2 x_{0}} \int_{-\infty}^{0} d x e^{(4+i k) x} \sum_{n=0}^{\infty} e^{4 n\left(x-x_{0}\right)} .
$$

Clearly, the series is uniformly convergent so the above relation becomes

$$
S_{0}=\frac{1}{\sinh 2 x_{0}}-2(2+i k) e^{-2 x_{0}} \sum_{n=0}^{\infty} e^{-4 n x_{0}} \int_{-\infty}^{0} d x e^{(4+4 n+i k) x}
$$

After integration over $x$, we obtain

$$
S_{0}=\frac{1}{\sinh 2 x_{0}}+2 i(2+i k) e^{-2 x_{0}} \sum_{n=0}^{\infty} \frac{e^{-4 n x_{0}}}{k-(4+4 n) i} .
$$

On the other hand, the above infinite series is a hypergeometric function. That is

$$
\sum_{n=0}^{\infty} \frac{e^{-4 n x_{0}}}{k-i(4+4 n)}=\frac{F\left(1, \frac{i}{4} k+1, \frac{i}{4} k+2, e^{-4 x_{0}}\right)}{k-4 i}
$$


Therefore, we get the following relation

$$
\begin{aligned}
S_{0}= & \frac{1}{\sinh 2 x_{0}} \\
& -2 \frac{k-2 i}{k-4 i} e^{-2 x_{0}} F\left(1, \frac{i}{4} k+1, \frac{i}{4} k+2, e^{-4 x_{0}}\right) .
\end{aligned}
$$

The hypergeometric function is defined by 25

$$
F(a, b, c, z)=\sum_{n=0}^{\infty} \frac{(a)_{n}(b)_{n}}{(c)_{n}} z^{n} \quad c \neq 0,-1,-2, \ldots
$$

where

$$
(a)_{n}=\frac{\Gamma(a+n)}{\Gamma(a)}=a(a+1) \ldots(a+n-1) \quad n=1,2,3, \ldots .
$$

The above series defines a function which is analytic when $|z|<1$. Also, the derivatives of the hypergeometric function are given by

$$
\frac{d^{n}}{d z^{n}} F(a, b, c, z)=\frac{(a)_{n}(b)_{n}}{(c)_{n}} F(a+n, b+n, c+n, z) .
$$

Next, for $n=1$ using (A.2) for the bulk three point coupling we have

$$
S_{1}=2 \int_{-\infty}^{0} d x e^{(2+i k) x} \cosh 2\left(x-x_{0}\right) \operatorname{coth} 2\left(x-x_{0}\right)\left(\operatorname{coth}^{2} 2\left(x-x_{0}\right)-1\right) .
$$

On the other hand, if we differentiate the left hand side of (A.10) with respect to $x_{0}$, which is given by

$$
\begin{array}{rl}
\frac{\partial S_{0}}{\partial x_{0}}=-\int_{-\infty}^{0} & d x e^{(2+i k) x}\left(4 \sinh 2\left(x-x_{0}\right)\left(\operatorname{coth}^{2} 2\left(x-x_{0}\right)-1\right)\right. \\
& \left.+8 \cosh 2\left(x-x_{0}\right) \operatorname{coth} 2\left(x-x_{0}\right)\left(1-\operatorname{coth}^{2} 2\left(x-x_{0}\right)\right)\right)
\end{array}
$$

and by comparing the above formula with (A.14), then the following equation may be derived

$$
S_{1}=\frac{1}{4} \frac{\partial S_{0}}{\partial x_{0}}+\int_{-\infty}^{0} d x e^{(2+i k) x} \frac{1}{\sinh 2\left(x-x_{0}\right)} .
$$

The second term in the above relation can be manipulated as before. Moreover, it is evident that we need to differentiate the right hand side of (A.10) which is equal to

$$
\begin{aligned}
\frac{\partial S_{0}}{\partial x_{0}}= & -\frac{2 \cosh 2 x_{0}}{\sinh ^{2} 2 x_{0}} \\
& +4 \frac{k-2 i}{k-4 i} e^{-2 x_{0}} F\left(1, \frac{i}{4} k+1, \frac{i}{4} k+2, e^{-4 x_{0}}\right) \\
& +8 \frac{k-2 i}{k-8 i} e^{-6 x_{0}} F\left(2, \frac{i}{4} k+2, \frac{i}{4} k+3, e^{-4 x_{0}}\right) .
\end{aligned}
$$


Finally, by substituting the relation (A.17) in (A.16), doing the computation of second term in the right-hand side of (A.16) and after simplifying we obtain

$$
\begin{aligned}
S_{1}= & -\frac{\cosh 2 x_{0}}{2 \sinh ^{2} 2 x_{0}} \\
& +\frac{k}{k-4 i} e^{-2 x_{0}} F\left(1, \frac{i}{4} k+1, \frac{i}{4} k+2, e^{-4 x_{0}}\right) \\
& +2 \frac{k-2 i}{k-8 i} e^{-6 x_{0}} F\left(2, \frac{i}{4} k+2, \frac{i}{4} k+3, e^{-4 x_{0}}\right) .
\end{aligned}
$$

In the same way, we may derive (A.1) when $\mathrm{n}$ is equal to 2 or 3 , however gradually the calculations become lengthy and we only write down the results, that is

$$
\begin{aligned}
S_{2}= & \frac{2}{3} \frac{1}{\sinh 2 x_{0}}+\frac{1}{6} \frac{\cosh ^{2} 2 x_{0}+1}{\sinh ^{3} 2 x_{0}} \\
& -\frac{1}{3} \frac{5 k-8 i}{k-4 i} e^{-2 x_{0}} F\left(1, \frac{i}{4} k+1, \frac{i}{4} k+2, e^{-4 x_{0}}\right) \\
& -\frac{4}{3} \frac{2 k-3 i}{k-8 i} e^{-6 x_{0}} F\left(2, \frac{i}{4} k+2, \frac{i}{4}\left(k+3, e^{-4 x_{0}}\right)\right. \\
& -\frac{8}{3} \frac{k-2 i}{k-12 i} e^{-10 x_{0}} F\left(3, \frac{i}{4} k+3, \frac{i}{4} k+4, e^{-4 x_{0}}\right)
\end{aligned}
$$

and

$$
\begin{aligned}
S_{3}= & -\frac{13}{24} \frac{\cosh 2 x_{0}}{\sinh ^{2} 2 x_{0}}-\frac{1}{24} \frac{\cosh ^{3} 2 x_{0}+5 \cosh 2 x_{0}}{\sinh ^{4} 2 x_{0}} \\
& +\frac{1}{6} \frac{7 k-4 i}{k-4 i} e^{-2 x_{0}} F\left(1, \frac{i}{4} k+1, \frac{i}{4} k+2, e^{-4 x_{0}}\right) \\
& +\frac{1}{3} \frac{13 k-22 i}{k-8 i} e^{-6 x_{0}} F\left(2, \frac{i}{4} k+2, \frac{i}{4} k+3, e^{-4 x_{0}}\right) \\
& +\frac{1}{3} \frac{18 k-32 i}{k-12 i} e^{-10 x_{0}} F\left(3, \frac{i}{4} k+3, \frac{i}{4} k+4, e^{-4 x_{0}}\right) \\
& +4 \frac{k-2 i}{k-16 i} e^{-14 x_{0}} F\left(4, \frac{i}{4} k+4, \frac{i}{4} k+5, e^{-4 x_{0}}\right) .
\end{aligned}
$$

\section{Appendix B}

In this Appendix we find the following integrals

$$
C_{n}=\int_{-\infty}^{0} d x e^{i k x} \cosh \left(\sqrt{2} \beta \phi_{0}\right) \operatorname{coth}^{n} 2\left(x-x_{0}\right) .
$$

Here, $\cosh \left(\sqrt{2} \beta \phi_{0}\right)$ is proportional to the bulk four point coupling and is given by

$$
\cosh \left(\sqrt{2} \beta \phi_{0}\right)=\left(2 \operatorname{coth}^{2} 2\left(x-x_{0}\right)-1\right) .
$$


So, let us calculate such integrals

$$
I_{n}=\int_{-\infty}^{0} d x e^{i k x} \operatorname{coth}^{n} 2\left(x-x_{0}\right)
$$

where $n=1,2, \ldots, 6$. It is better to find the solution of the above integrals when $n=1$. Considering the following inequality (see Appendix A)

$$
0<e^{4\left(x-x_{0}\right)}<1
$$

and therefore, in what follows we will use the expanded form of coth $2\left(x-x_{0}\right)$ as

$$
\operatorname{coth} 2\left(x-x_{0}\right)=1-2 \sum_{n=0}^{\infty} e^{4 n\left(x-x_{0}\right)} .
$$

It turns out to be simple if we consider this integral

$$
\int_{-\infty}^{0} d x e^{(\tau+i k) x} \operatorname{coth} 2\left(x-x_{0}\right)
$$

where $\tau$ is a positive constant quantity which will be taken to zero at the end of the calculation. Moreover, by using (B.4), then (B.5) becomes

$$
\int_{-\infty}^{0} d x e^{(\tau+i k) x}-2 \int_{-\infty}^{0} d x \sum_{n=0}^{\infty} e^{4 n\left(x-x_{0}\right)} e^{(\tau+i k) x}
$$

and regarding the arguments in Appendix A, we may evaluate the above relation to obtain

$$
-\frac{i}{k-i \tau}+2 \sum_{n=0}^{\infty} \frac{i}{k-(\tau+4 n) i} e^{-4 n x_{0}} .
$$

Now, we can write down the desired result, that is,

$$
I_{1}=-\frac{i}{k}+2 i \sum_{n=0}^{\infty} \frac{e^{-4 n x_{0}}}{k-4 n i} .
$$

On the other hand, the above series is equal to a hypergeometric function

$$
\sum_{n=0}^{\infty} \frac{e^{-4 n x_{0}}}{k-4 n i}=\frac{1}{k} F\left(1, \frac{i}{4} k, \frac{i}{4} k+1, e^{-4 x_{0}}\right)
$$

and finally we find this formula

$$
I_{1}=-\frac{i}{k}+\frac{2 i}{k} F\left(1, \frac{i}{4} k, \frac{i}{4} k+1, e^{-4 x_{0}}\right) .
$$

Now, let us compute (B.3) when $n=2$ and in order to solve it, it is sufficient to differentiate both sides of $(\bar{B} .10)$ with respect to $x_{0}$ to obtain

$$
I_{2}=-\frac{i}{k}-\frac{4 i}{k-4 i} e^{-4 x_{0}} F\left(2, \frac{i}{4} k+1, \frac{i}{4} k+2, e^{-4 x_{0}}\right) .
$$


We can follow a similar method to get higher order forms of (B.3) which we need in this paper so, it is appropriate to write down all of them i.e.

$$
\begin{aligned}
& I_{3}=-\frac{i}{k}+\frac{2 i}{k} F\left(1, \frac{i}{4} k, \frac{i}{4} k+1, e^{-4 x_{0}}\right) \\
& \frac{4 i}{k-4 i} e^{-4 x_{0}} F\left(2, \frac{i}{4} k+1, \frac{i}{4} k+2, e^{-4 x_{0}}\right) \\
& \frac{8 i}{k-8 i} e^{-8 x_{0}} F\left(3, \frac{i}{4} k+2, \frac{i}{4} k+3, e^{-4 x_{0}}\right) \text {, } \\
& I_{4}=-\frac{i}{k}-\frac{8 i}{k-4 i} e^{-4 x_{0}} F\left(2, \frac{i}{4} k+1, \frac{i}{4} k+2, e^{-4 x_{0}}\right) \\
& -\frac{16 i}{k-8 i} e^{-8 x_{0}} F\left(3, \frac{i}{4} k+2, \frac{i}{4} k+3, e^{-4 x_{0}}\right) \\
& -\frac{16 i}{k-12 i} e^{-12 x_{0}} F\left(4, \frac{i}{4} k+3, \frac{i}{4} k+4, e^{-4 x_{0}}\right) \text {, } \\
& I_{5}=-\frac{i}{k}+\frac{2 i}{k} F\left(1, \frac{i}{4} k, \frac{i}{4} k+1, e^{-4 x_{0}}\right) \\
& \frac{8 i}{k-4 i} e^{-4 x_{0}} F\left(2, \frac{i}{4} k+1, \frac{i}{4} k+2, e^{-4 x_{0}}\right) \\
& \frac{32 i}{k-8 i} e^{-8 x_{0}} F\left(3, \frac{i}{4} k+2, \frac{i}{4} k+3, e^{-4 x_{0}}\right) \\
& \frac{48 i}{k-12 i} e^{-12 x_{0}} F\left(4, \frac{i}{4} k+3, \frac{i}{4} k+4, e^{-4 x_{0}}\right) \\
& \frac{32 i}{k-16 i} e^{-16 x_{0}} F\left(5, \frac{i}{4} k+4, \frac{i}{4} k+5, e^{-4 x_{0}}\right)
\end{aligned}
$$

and

$$
\begin{aligned}
I_{6}= & -\frac{i}{k}-\frac{12 i}{k-4 i} e^{-4 x_{0}} F\left(2, \frac{i}{4} k+1, \frac{i}{4} k+2, e^{-4 x_{0}}\right) \\
& -\frac{48 i}{k-8 i} e^{-8 x_{0}} F\left(3, \frac{i}{4} k+2, \frac{i}{4} k+3, e^{-4 x_{0}}\right) \\
& -\frac{112 i}{k-12 i} e^{-12 x_{0}} F\left(4, \frac{i}{4} k+3, \frac{i}{4} k+4, e^{-4 x_{0}}\right) \\
& -\frac{128 i}{k-16 i} e^{-16 x_{0}} F\left(5, \frac{i}{4} k+4, \frac{i}{4} k+5, e^{-4 x_{0}}\right) \\
& -\frac{64 i}{k-20 i} e^{-20 x_{0}} F\left(6, \frac{i}{4} k+5, \frac{i}{4} k+6, e^{-4 x_{0}}\right) .
\end{aligned}
$$

\section{References}

[1] A.E. Arinshtein, V.A. Fateev and A.B. Zamolodchikov, 'Quantum S-matrix of the 1+1 dimensional Toda chain', Phys.Lett. B87 (1979) 389-392. 
[2] A.V. Mikhailov, M.A. Olshanetsky and A.M. Perelomov, 'Tow-dimensional generalized Toda lattice', Comm.Math.Phys. 79 (1981)43

[3] E. Corrigan, 'Recent developments in affine Toda quantum field theory', CRMCAP Summer School on Particles and Fields '94, Banff, eds. G. Semenoff, L. Vinet (Springer New York) 1999, hep-th/9412213

[4] E. Corrigan, P.E. Dorey, R.H. Rietdijk and R. Sasaki, ' Affine Toda field theory on a half-line' Phys.Lett. B333 (1994) 83.

[5] E. Corrigan, P.E. Dorey, R.H. Rietdijk, 'Aspects of affine Toda field theory on a half-line', Prog.Theor.Phys.Suppl. 118:143-164, 1995, hep-th/9407148.

[6] P. Bowcock, E. Corrigan, P.E. Dorey and R.H. Rietdijk, 'Classically integrable boundary conditions for affine Toda field theory', Nucl.Phys B445 (1995) 469.

[7] A. Fujii and R. Sasaki, 'Boundary effects in integrable field theory on a half-line', Prog.Theor.Phys. 93:1123-1134, 1995, hep-th/9503083.

[8] A. Fring and R. Koberle, 'Factorized scattering in the presence of reflecting boundaries', Nucl.Phys. B421 (1994) 159.

[9] A. Fring and R. Koberle, 'Affine Toda field theory in the presence of reflecting boundaries', Nucl.Phys. B419 (1994) 647.

[10] G.M. Gandenberger, 'On $a_{2}^{(1)}$ reflection matrices and affine Toda theories', Nucl. Phys. B542:659-693,1999, hep-th/9806003

[11] G.W. Delius and G.M. Gandenberger, 'Particle reflection amplitudes in $a_{n}^{(1)}$ Toda field theories', Nucl. Phys. B554:325-364,1999, hep-th/9904002

[12] M. Perkins and P. Bowcock, 'Quantum corrections to the classical reflection factor in $a_{2}^{(1)}$ Toda field theory', Nucl.Phys. B538 (1999) 612-630.

[13] E. Corrigan and G.W. Delius, 'Boundary breathers in the sinh-Gordon model', J.Phys. A32:8601-8614, 1999, hep-th/9909145

[14] E. Corrigan, 'On duality and reflection factors for the sinh-Gordon model with a boundary', Int.J.Mod.Phys. A13:2709-2722, 1998, hep-th/9707235.

[15] J.D. Kim, 'Boundary reflection matrix in perturbative quantum field theory', Phys.Lett. B353 (1995) 213.

[16] J.D. Kim, 'Boundary reflection matrix for A-D-E affine Toda field theory', hepth/9506031.

[17] N. Topor, 'Perturbation method for boundary S-matrix in 2D quantum field theory', Mod.Phys.Lett. A12 (1997) 2951-2962.

[18] H.W. Braden, E. Corrigan, P.E. Dorey and R. Sasaki, 'Affine Toda field theory and exact S-matrices', Nucl. Phys. B338 :689-746,1990.

[19] S. Ghoshal, 'Boundary state boundary S-matrix of the sine-Gordon model', Int.J.Mod.Phys. A9 (1994) 4801. 
[20] S. Ghoshal and A. Zamolodchikov, 'Boundary S matrix and boundary state in two-dimensional integrable quantum field theory, Int.J.Mod.Phys. A9 (1994) 3841.

[21] A. Chenaghlou and E. Corrigan, 'First order quantum corrections to the classical reflection factor of the sinh-Gordon model', hep-th/0002065.

[22] L.D. Faddeev and V.E. Korepin, 'Quantum theory of solitons, Phys.Rep. 42 (1978) $1-87$.

[23] A.B. Zamolodchikov and Al.B. Zamolodchikov, 'Factorized S-matrices in two dimensions as the exact solutions of certain relativistic quantum field theory models' Ann.Phys. 120 (1979) 253-291.

[24] M. Ablikim, 'Boundary sinh-Gordon model and its supersymmetric extension', Ph.D thesis, University of Durham, Dec. 1999.

[25] G. Arfken, Mathematical Methods for Physicists, Academic Press, Inc., 1985. 